\title{
A Bridge between Quantum Mechanics and Astronomy
}

\author{
Anna C.M. Backerra ${ }^{1}$ \\ ${ }^{1}$ Gualtherus Sylvanusstraat 2, 7412 DM Deventer, The Netherlands \\ Correspondence: Anna C.M. Backerra, Gualtherus Sylvanusstraat 2, 7412 DM Deventer, The Netherlands. \\ E-mail: annabackerra@gmail.com
}

$\begin{aligned} & \text { Received: November 19, } 2015 \\ & \text { Accepted: December 2, } 2015 \quad \text { Online Published: December 22, } 2015 \\ & \text { doi:10.5539/apr.v8n1p16 }\end{aligned} \quad$ URL: http://dx.doi.org/10.5539/apr.v8n1p16

\begin{abstract}
Small-scale physics called quantum mechanics, is still incompatible with large-scale physics as developed by Einstein in his general relativity theory. By using twin physics, which is a dualistic way of considering the universe, and following Einstein's later advice it is possible to create a bridge between these extremes. The formulation is carried out using complementary language in which time and space necessarily occur as two distinct qualities, although they are treated analogously. The basic item in the theory is the Heisenberg unit, which has a constant amount of potential energy, and which is supplied with mathematical attributes; by interaction with another Heisenberg unit, these attributes are transformed into physical qualities. With this theory, a photon can be described such that its velocity is constant without using the related postulate, showing how the speed of light is the link between small- and large-scale physics. The Planck constant emerges from the explanation. The photon is accompanied by a so-called anti-photon, being a charged, massless particle, traveling with the same velocity and exchanging electromagnetic energy.
\end{abstract}

Keywords: complementarity, electromagnetism, Planck constant, photon, quantum mechanics, velocity of light

\section{Introduction}

Our theory originates in experiments (White, 1964) from 1927 showing that matter can appear in two distinct ways, as a determined "particle" and as a so-called "wave", whose position is uncertain. This dualism was the start of quantum mechanics. The existence of fundamental uncertainty was a shock to the scientific world, which, since Newton proposed the theory of mechanics in his "Principia" in 1687, had been ruled by the conviction that nature was deterministically organized. Objects were supposed to have mass, and to be existing in an infinite empty space which was supposed to have no energy, and thus space was ignored in science.

The first way of dealing with these experimental results was the Copenhagen interpretation, a collection of axioms or doctrines largely devised in the years 1925 - 1927 by Niels Bohr and Werner Heisenberg (Heisenberg, 1971). In their discussions, Bohr was convinced that a philosophical comprehension of a physical problem had to be completely explained before being able to describe it mathematically. He introduced the notion "complementarity" as a way of making the duality between "particles" and "waves" the starting point for a different interpretation, but at the time was not successful in finding a mathematical basis for it. Later Heisenberg too was convinced that this was the right direction in principle, and wrote two books about the philosophical consequences of this interpretation (Heisenberg, 1930/1949, 1971).

Bohr and Heisenberg tried to reconcile uncertainty with determinism in a manner conducive to human understanding by considering subatomic events as indeterministic discontinuous transitions between discrete stationary states. Incompatible conjugate properties at that scale were not supposed to be measurable with arbitrary precision at the same time. This is expressed in detail as Heisenberg's uncertainty principle which is a special case of a more general complementarity proposed by Bohr. Although this principle is still accepted, the Copenhagen interpretation did not lead to a complete, satisfying description of nature that offered a place to this behavior of matter at a subatomic scale, as well as to gravitational behavior at an astronomic scale, as described by Einstein's general theory of relativity.

Many alternative theories arose during the course of the $20^{\text {th }}$ century such as the Statistical Interpretation (Messiah, 1961), the Many Worlds Theory (Vaidman, 2002) and Hidden Variables (Rietdijk, 2003). They all were characterized by a continuation of the belief in determinism. None of them could create a bridge between the small-scale world, which seemed to be ruled by probability, and the large-scale world, which seemed to obey 
Einstein's relativity theory. However, among each generation of physicists, a few continued to search for the possibility of accepting duality as fundamental in nature, including Heisenberg (Heisenberg, 1930/1949, 1971), Von Weizsäcker (Weizsäcker, 1941, 1955) and Jammer (Jammer, 1974, 2000). They realized that the available mathematics had been developed to serve only deterministic science and although their work did not result in a complete theory, it contains useful advice about how to construct new mathematics suited to describing physical phenomena including uncertainty.

We continued this development first by expressing the Heisenberg uncertainty principle in general terms of duality as "each observation of certainty implies an amount of uncertainty". Then we supposed that any two basic concepts of duality are of equal importance as suggested by Bohr (see Heisenberg, 1971) which implies that the mirrored case also has to be true: "each observation of uncertainty implies an amount of certainty". Thus we extended the Heisenberg uncertainty principle. From that starting point, we gathered the advice of these predecessors to construct a mathematical method in which uncertainty is interpreted as an independent concept in nature and called this method "complementary language".

In addition to this, we followed the advice of Albert Einstein (1996/1936-1950) for the construction of a new physical theory, written from 1936 to 1950. Although he was a confirmed opponent of any theory with even a trace of indeterminism in it, he understood very well that there was a large problem in physics due to the incompatibility of large- and small-scale theories. He presented a general method for the construction of a completely new, sound physical theory in three steps, thus suggesting that this could be necessary in the future. The first step is to find a two-sided concept of a physical object. On the one hand this concept is an arbitrary creation of the brain, and on the other hand it derives its meaning and vindication only from the entire sensory perception with which we associate it. The second step is to give this concept a meaning independent of sensory perceptions, stating that the connection between elementary concepts and groups of perceptions can only be understood in an intuitive way. The third step is just to put some rules, which might be definitions as well as laws of nature, depending on the chosen representation, and that completes the process. With this conception of a physical theory in general, he stressed the necessity of connecting the basics of any physical theory to human intuition. This refers to the origin of physics, rooted in the combination of facts and philosophy. However, in the development of physical theories after Einstein, less and less attention was paid to human perception, let alone to intuition. However, we took his advice in making our choice of mathematical items.

Einstein offered us more stepping stones for the development of twin physics. We also used the basics of his special relativity theory, also published in 1905 (Einstein, 1905a), in which he elaborates upon the principle proposed by Mach (1838-1916) on relativity, saying that an independent grid of coordinates as a frame of reference does not exist. Although Einstein still used a resting coordinate frame, he required the energetic states of physical systems to be independent of it. This requirement is anchored in our theory by the introduction of "Heisenberg units" (H-units) as elementary units of potential energy, being able to manifest themselves by interacting with each other and so generating phenomena called Heisenberg events. Because H-units are defined without a frame of reference, they can move only with respect to each other. Apart from this, we do not use Einstein's postulate that light in vacuum has a constant velocity, which appeared in the same paper, because we can derive this without using a postulate.

As the last point of agreement, we incorporated Einstein's discovery, also written in 1905, that mass and electromagnetic energy are equivalent (Einstein, 1905b), by supposing that interacting H-units are the one and only energetic source of all physical phenomena, meaningboth mass and space, together with all their features and electromagnetic items.

However, there are two aspects in which we deviate from Einstein's ideas, both being the consequence of considering uncertainty as a basic, independent aspect of nature. The first aspect is that we do not consider time to be a determinate physical item, so each H-unit is supplied with determinate as well as indeterminate time attributes. In a special edition of the journal "Scientific American" (Scientific American, 2014) well-known physicists have written abundantly about the human tendency to consider time in another way, as we are used to doing in classical as well as in modern physics. Many of them testify to the irrepressible desire to introduce uncertainty into the concept of time. Two quotations are: "All these more or less magic indications of the flow of time can be considered as primitive ways to catch the indeterminate aspect of time," and "The best thing [scientists and philosophers] can say is, that time is an extra dimension akin (but not identical) to space". Our choice of time attributes meets this tendency. A third quotation is: "The past we think of as having slipped out of existence" can also be found back in twin theory, because it does not incorporate the past. 
The second aspect in which we deviate from the ideas of Einstein can be found in his general relativity theory (Einstein, 1916) in which he describes special relativity in a way, published by Hermann Minkowski in 1907 as "Raum und Zeit" (see Corry, 1998). This mathematician supposed the formal equivalence of space-like and time-like coordinates. Minkowski based this upon the work of Henri Poincaré, who in 1906 published a way of explaining Lorentz transformations in terms of the familiar Euclidean rotation, by taking (imaginary) time as the fourth dimension. Minkowski used a real time coordinate instead of an imaginary one and reformulated the then recent theory of special relativity in four dimensions. Einstein embraced this method and managed to describe a gravitational field with it. However, we do not use time and space as a four-dimensional continuum, because it is not suited to describing time in an indeterminate way. As we explained in our previous paper, gravity can be described by twin physics without making use of the Minkowski space. On the other hand, to deviate as little as possible from Einstein's theory, we treat space and time mathematically in the same way; the only difference is in the dimensions, space being three-dimensional and time one-dimensional.

After having explained which choices we made with respect to the history of physics, we are ready to unfold our theory. In order to be able to grasp its reach, we recommended bearing three of its essential features in mind. The first essential feature is the supposed complementary duality in all aspects of nature, such that the two opposite concepts are of equivalent importance. In our theory we consider mass and space as complementary, so space is not considered as "nothing", indicating a lack of matter, but as an independent energetic physical object. Time is not only described by determinate attributes, but also with indeterminate ones which are as important. Most of us are not familiar with the notion of complementary duality, although there are many examples in daily life. A non-parallel beam of light can be convergent or divergent, producing different physical effects. A source of water pouring out into the total surroundings, has a well as its complement gathering it. An anode repels electrons, a cathode attracts them. Complementarity can also be experienced as a mental attitude, concentrated or expanded, the first being necessary to read a paper like this, the second to look after seven children playing in a park. The second essential feature of our theory is the assumption that potential energy exists in elementary units, called Heisenberg units. Because potential energy principally cannot be observed, this is a mathematical starting point. Only after transforming the mathematical result of interaction between Heisenberg units into actual energetic items can descriptions of physical observations be obtained. The third essential feature is that all mathematical items, after being transformed into physical items, can only play either a major or minor role in an observation, nothing in between.

The mathematical method called complementary language that we developed in previous papers (Backerra, 2010, 2012, 2014), is based upon the using the theory of sets. The H-unit is provided with a set of complementary attributes which can be associated with time, space and mark (the mathematical precursor of electromagnetic features), for instance a point, a finite three-dimensional space, a number or a vector field. These attributes are chosen to be as close as possible to human perceptions in daily life. This requires imagination and creativity because we are not used to describing basic physical items in determinate as well as indeterminate way and as being of major as well as minor importance. In this way the influence of all of the relevant items of the H-units can be followed separately, whether they are of major or minor importance, in a determinate or indeterminate way. Also it is possible to construct rules for the interaction of two H-units. This will be shown in the next section. The mathematical result of the interaction is a set of four elements called a zipper, containing many variables depending on the relative positions of the Heisenberg units, which fortunately decrease, usually drastically. After transformation into actual energetic items, they can be identified as many well-known physical observations.

In this paper we search for the description of a photon and thus focus upon massless physical items. To aid comprehension, we begin by explaining the theory as briefly as possible and continue with the simplest example of two coinciding $\mathrm{H}$-units generating a mass, in order for readers to become acquainted with complementary language and its application in twin physics.

It is useful to keep in mind our energetic definitions of mass and space (Backerra, 2012). We have introduced the combined expression "energetic space" for any appearing timespace object and have distinguished mass and space according to the concentration of energy: Mass has an extremely high concentration of energy whereas space has an extremely low energy concentration and is even considered to be zero. These two distinct events are called 'compact space' and 'extended space' respectively; in this paper, just for the sake of convenience, we express them as the classical terms 'mass' and 'space'.

In previous papers we supposed that our method incorporated Planck's quantization from scatch, but this turned out to be an error, evoked by the introduction of an H-unit with a constant amount of potential energy. In this paper, quantization turns out to be not the origin, but a consequence of our theory, so we do not need this 
supposition. Another change is the addition of a tilde above mathematical items, which disappears after the items are transformed into physical items. Furthermore some other small improvements there will be made and these will be indicated at the appropriate places.

\section{Complementary Language in General}

We constructed complementary language using the definition of Max Jammer (Jammer, 1974), in which he states that a complementary interpretation has to satisfy the following conditions: (a) it contains at least two descriptions $A_{1}$ and $A_{2}$ of its substance-matter; (b) $A_{1}$ and $A_{2}$ refer to the same universe of discourse; (c) neither $A_{1}$ nor $A_{2}$, if taken alone, accounts exhaustively for all phenomena of this universe; (d) $A_{1}$ and $A_{2}$ are mutually exclusive in the sense that their combination into a single description would lead to logical contradictions."

Besides of that, we need some basic items as representatives for all phenomena in the world. In the past it appeared that one mass-carrying building block of nature is not existing; the amount of so-called elementary particles is impressive large. A solution for that problem was found in the definition of quarks as building blocks of elementary particles, existing in much less variants and by definition impossibly to isolate. By this step a more abstract way of thinking about the basics of nature was introduced and nowadays many scientists believe in their existence.

We go one step further by introducing a completely abstract item as basic in nature: the Heisenberg unit (H-unit) as an elementary unity of potential energy. In that way we build our theory upon the most important concept in physics, which is the conservation of energy, combining even mass and light. There exists no minimum amount of energy; the only item which comes close to it is $h$, Planck's constant, but its dimension is Joule.second. Although the concept of actual energy is not supplied with an elementary amount, there is no objection to introduce an elementary amount of potential energy. We are already acquainted with the abstract character of potential energy, being impossible to measure as long as it is not transformed into actual energy. For instance, the amount of potential gravitational energy of an object close to earth can only be measured by allowing the object to fall down. Clearly transformation from potential to actual energy happens only if there is a relevant interaction between the observed item with its surrounding. Thus potential energy can be defined as the power to interact. This feature has to be incorporated into the definition of the $\mathrm{H}$-unit.

Thus we define: An $\boldsymbol{H}$-unit is an elementary unity of potential energy, expressed in complementary terms; it can only manifest itself by interacting with other ones. The potential energy of interacting H-units is converted into phenomena, called H-events, having actual energy. We suppose that this conversion occurs in a complementary way, which seems to be a natural step in a theory based upon complementarities. Because no independent frame of reference is used, any movement of an H-event is only possible with respect to another H-unit, so relativity is incorporated from scratch. An H-unit is supplied with pairs of complementary mathematical attributes, each pair containing a determined and indetermined attribute. In doing so, the extended Heisenberg's uncertainty principle is incorporated from scratch.

We introduced three qualities to which attributes can belong: space, time and mark (the mathematical precursor of electromagnetic features). Therefore an $\mathrm{H}$-unit $H_{i}$ possesses for each quality a set $h_{i}$ of mathematical attributes in two types: determinate attributes represented by $D_{i}$ or $d_{i}$, and indeterminate ones represented by $U^{i}$ or $u^{i} . D$ and $d$ represent 'Determinate'; $U$ and $u$ represent "uncertain" as one of the possible circumscriptions of indeterminism. The difference between capitals and lower case will become clear later. They are collected in the general set of attributes

$$
h_{i}=\left\{D_{i}, U^{i}, d_{i}, u^{i}\right\}
$$

Interaction between two H-units is based upon the exchange of their attributes according to mathematical rules.

After transforming from mathematical into physical items, potential energy is transformed into actual energy, describing physical observations. Transformation of mathematical into physical items is a technique which was already used by Schrödinger in his famous equation (see Messiah, 1961), containing an imaginary part, disappearing at the end of the derivation, the so-called "collapse of the wave function". In that time it was experienced as a serious problem. It is the first example of an explicit transformation of mathematics into physics. We will use this technique again, this time without any problems.

Our way of dealing with phenomena is briefly called "twin physics". In an alternative way to our previous papers, we have developed a method to explain this new type of mathematics in a more accessible way, guided by a simple example and exemplified below. 


\section{Space Attributes}

For the deterministic space attribute we define mathematical point $\widetilde{P}_{i}$, the location of the H-unit, as its address. The tilde indicates that the item has no physical meaning. For the indeterminate attribute we define mathematical space $\tilde{S}^{i} \backslash \widetilde{P}_{i}$, the extensiveness of the H-unit in which $\tilde{S}^{i}$ is a finite sphere with central point $\widetilde{P}_{i}$, having a real, large but not infine radius $R$, the border excluded. The magnitude of this sphere might be arbitrary large, even of astronomic size. These attributes are complementary with respect to each other and together they constitute a complete geometric system, which is $\tilde{S}^{i}$. Then a set of space attributes $h_{i}(\tilde{\mathbf{x}})$ of an H-unit $H_{i}$ can be defined as

$$
h_{i}(\tilde{\mathbf{x}})=\left\{\widetilde{P}_{i}, \widetilde{S}^{i} \backslash \widetilde{P}_{i}\right\}
$$

in which $\tilde{S}_{\tilde{S}_{i}} \backslash \tilde{P}_{i}$ indicates a three-dimensional mathematical space. The two elements of this set are attributes $\widetilde{P}_{i}$ and $\tilde{S}_{i} \backslash \tilde{P}_{i}$, each describing a part of space $\tilde{S}^{i}$. Lower indices of the attributes indicate determinate and upper indices indeterminate attributes. Point $\widetilde{P}_{i}$ is the most determinate spatial object we can choose. Space $\tilde{S}^{t}$ is the most indeterminate one. Each of its points has an indeterminate position, which is conceived as being stretched out over the entire space. Mind that this expression for $h_{i}(\tilde{\mathbf{x}})$ is only a starting-point; later we will add more attributes.

The interaction between H-units $H_{i}$ and $H_{j}$ is written as $h_{i}(\tilde{\mathbf{x}}) * h_{j}(\tilde{\mathbf{x}})$. We suppose that the potential energy of two H-units $H_{i}$ and $H_{j}$ will transform completely into actual energy if their space attributes coincide. Note that in physics two items without any difference cannot exist at the same place, but in mathematics this is no problem.

To combine attributes of two interacting H-units, we introduce the link operator $\propto$. The definition of $X \propto Y$ (pronounced as ' $X$ linked to $Y$ ') is, that mathematical objects $X$ and $Y$, after transformation into a physical space, occur combined in a physical observation. The link $X \propto Y$ is defined as their intersection. If $X \propto Y$ then $Y \propto X$; if $X=Y$ then $X \propto Y=X ; X \propto \varnothing=\varnothing$ in which $\varnothing$ is an empty set. With this definition the interaction between $\mathrm{H}$-units can be defined as the set of all possible links of their attributes.

As an example we consider the interaction between two coinciding H-units, with $\widetilde{P}_{i}=\widetilde{P}_{j}=\widetilde{P}$ and $\widetilde{S}^{i}=\widetilde{S}^{j}=\widetilde{S}$. Because in that case $\widetilde{P}_{i} \notin \widetilde{S}^{j}$ and $\widetilde{P}_{j} \notin \widetilde{S}^{i}$, is $\widetilde{P}_{i} \propto\left(\widetilde{S}^{j} \backslash \widetilde{P}_{j}\right)=\widetilde{P}_{j} \propto\left(\widetilde{S}^{i} \backslash \widetilde{P}_{i}\right)=\varnothing$. Then the interaction between $H_{i}$ and $H_{j}$ can be described by the set of the only two links which can be constructed, which is

$$
h_{i}(\tilde{\mathbf{x}}) * h_{j}(\tilde{\mathbf{x}})=\left\{\widetilde{P}_{i} \propto \widetilde{P}_{j}, \widetilde{S}^{i} \backslash \widetilde{P}_{i} \propto \widetilde{S}^{j} \backslash \widetilde{P}_{j}\right\}=\{\widetilde{P}, \tilde{S} \backslash \widetilde{P}\} .
$$

The elements $\widetilde{P}$ and $\tilde{S} \backslash \widetilde{P}$ of this new set are exchanged attributes, instead of the single attributes of one H-unit as we saw in formula (2). The lack of indices indicates that something is changed.

The transformation of a mathematical spatial object into a physical spatial observation is defined as an operation which changes the mathematical object into a physical object and ascribes actual space energy to it. This will be indicated by removing the tilde. We assume that the spatial energy of an object is proportional to the size of its three-dimensional space. The transformation of mathematical element $\tilde{X}$ into a physical observation is written as $[\tilde{X}]$. The transformation of mathematical point $\widetilde{P}$ into a physical space is defined by $[P]=P$ with $P$ being a physical point, containing no energy because it has no spatial extension. The transformation of a mathematical space $\tilde{S} \backslash \tilde{P}$ into a physical space is defined by $[\tilde{S} \backslash \tilde{P}]=S \backslash P$, with $S \backslash P$ being a large physical space, containing energy.

We collect all possible observations, resulting from the interaction between two H-units, in the set of observations $O(\mathbf{x})$. In our example of coinciding H-units, this set is (using Equation (3)):

$$
O(\mathbf{x})=\left[h_{i}(\tilde{\mathbf{x}}) * h_{j}(\tilde{\mathbf{x}})\right]=\left\{\left[\widetilde{P}_{i}\right],\left[\tilde{S}^{i} \backslash \widetilde{P}_{i}\right]\right\},
$$

so, after carrying out the transformations, the set of observations can be written as

$$
O(\mathbf{x})=\left\{O_{1}, O_{2}\right\}=\{P, S \backslash P\}
$$

The set contains two elements, which are two possible observations: point of space $P$ and space $S \backslash P$. These physical objects are extreme opposites, as well in geometric as in energetic sense. Because the point carries no 
energy at all, the space carries all actual energy and as the H-units coincide, this is equal to the sum of potential energy of two H-units.

The concept of energetic space meets our experience, because experimentally it is well known that empty space can produce particles spontaneously. The problem is in point $P$, which cannot be a description of mass because it cannot store energy. Clearly we have to expand our description such, that the point gets the disposal of a spatial extension.

To add an extra attribute to the H-unit,,$_{i}$ we introduce small sphere $\tilde{S}^{i}$ with central point $\tilde{P}_{i}$, having radius $r<<R$, its border excluded. We call $S$ the minor space and in contrast with that, $S \backslash \mathcal{P}_{i}$ is called the major space of the H-unit. Because we are restricted to complementary attributes, we have to define a second minor attribute, which has to be complementary to the minor space, excluding it and together constituting a complete space system. As an obvious choice we take the border of the minor space, being a two-dimensional mathematical object, called pellicle $p_{i}$, which can be imagined as the skin of an apple. Then the complete minor space system consists of the minor space and its border. We have to decide which one of these two extra attributes has to be considered as a determinate and which as an indeterminate minor attribute, with respect to each other. Because all points of the pellicle have a distance of exactly $r$ to $P_{i}$, the pellicle seems to be suited as a determinate attribute. The points of the minor space $S$ have a large variety of distances from $P_{i}$, reaching from zero up to $r$, the upper border excluded, which makes the minor space suited to act as an indeterminate attribute. To avoid dimensional problems after transforming into real three-dimensional space, we provide the pellicle with three instead of two dimensions, by allowing it to have an infinitesimally small width $d r$, with $d r<<r$. Thus the set of space attributes $h_{i}(\tilde{\mathbf{x}})$ of $H_{i}$ (see Equation (2)) is extended to a set of four elements

$$
h_{i}(\tilde{\mathbf{x}})=\left\{\widetilde{P}_{i}, \tilde{S}^{i} \backslash \widetilde{P}_{i}, \tilde{p}_{i}, \tilde{s}^{i}\right\},
$$

Capitals and small letters indicate major and minor attributes $s_{i}$ respectively. This set can be related to daily experience by imagining a house: $P_{i}$ represents its address, $S \backslash P_{i}$ the city where it is located, $p_{i}$ the outer wall and $s$ the inner space of the house. Because an H-unit is a unit of potential energy, we suppose for the moment that all attributes for two distinct $\mathrm{H}$-units are identical in size. Thus two $\mathrm{H}$-units $H_{i}$ and $H_{j}$ can differ merely in the position of their major points of space with respect to each other, which is indicated by their central points $\tilde{P}_{i}$ and $\widetilde{P}_{j}$.

Obviously the interaction of $\mathrm{H}$-units will be more complicated now. To obtain interaction rules for interacting major and minor objects, we will return to the extended Heisenberg's uncertainty principle, saying that each 'extreme certain observation' contains an 'extremely small influence of uncertainty', and, the other way round, each 'extreme uncertain observation' contains an 'extremely small influence of certainty'. This fundamental feature of nature can be anchored in our theory by supposing that all attributes add to any observation in pairs, which are restricted by the following axiom: A joined pair of attributes contributes to an observation in pairs, which are such that one member is of major and the other of minor importance. Note that the members of one pair may be both determinate, both indeterminate or mixed. A joined pair of attributes is in general written as $X \bowtie y$ (pronounced as " $X$ is joined with $y$ "), in which the attribute of major importance is indicated in capitals and that of minor importance in lower case. The join operator $\triangleright \triangleleft$ is defined such that the attributes $X$ and $y$, after having transformed them into a physical space, are necessarily observed together.

This axiom is the heart of our theory and it replaces the two axioms in our previous work. In that time we expected that even a single joined pair could produce an observation, which introduced the requirement that one attribute was determinate and the other indeterminate. Later we did not find any indication that a single joined pair after transformation describes any physical item. Thus a joined pair has to be combined with at least one other joined pair to obain any physical result, so the extended Heisenberg's uncertainty principle can be incorporated without the previous requirement and thus one axiom is dropped; in this paper it became clear that the previous two axioms even could introduce errors.

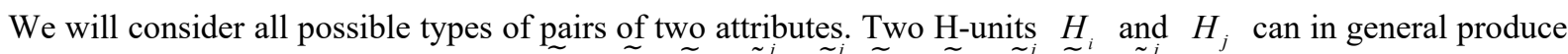
eight mixed pairs of attributes, being $\widetilde{P}_{i \triangleright \triangleleft} \widetilde{p}_{j}, \widetilde{P}_{i \triangleright \triangleleft} \tilde{S}^{j}, \widetilde{S}^{i} \backslash \widetilde{P}_{i \triangleright \triangleleft} \widetilde{p}_{i}, \widetilde{S}^{i} \backslash \widetilde{P}_{i \triangleright \triangleleft}^{i} \tilde{S}^{j}$ and four similar more, with

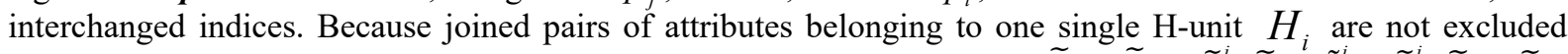
$\underset{\widetilde{S}^{i} \backslash \widetilde{P}}{\text { from }}$ the definition, we involve so-called self-pairs as well, being $\widetilde{P}_{i \triangleright \triangleleft} \widetilde{p}_{i}, \quad \widetilde{S}^{i} \backslash \widetilde{P}_{i \triangleright \triangleleft} \widetilde{s}^{i}, \quad \widetilde{S}^{i} \backslash \widetilde{P}_{i \triangleright \triangleleft} \widetilde{p}_{i}$, $\widetilde{S}^{i} \backslash \widetilde{P}_{i \triangleright \triangleleft} \tilde{S}^{i}$ and four similar more, having indices $j$. In total there exist 16 distinct joined pairs. 
Because we want to describe a particle, the definition of joined pairs has to be such that, after transformation, a small physical object having a spatial extension can be described. For that reason joined pair $\widetilde{P}_{i \triangleright \triangleleft} \tilde{S}^{j}$, as the first one, is defined as

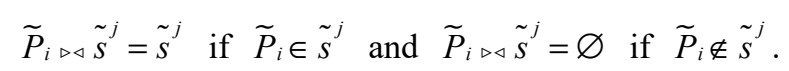

This definition says that, if the interaction allows a major point to be an element of a minor space (its own or the minor space of another H-unit), the point unites with the entire minor space. This definition of joining is simply extended to all remaining joined pairs containing a major point, so:

$$
\widetilde{P}_{i \triangleright \triangleleft} \tilde{p}_{j}=\tilde{p}_{j} \text { if } \widetilde{P}_{i} \in \tilde{p}_{j} ; \tilde{P}_{i \triangleright \triangleleft} \tilde{p}_{j}=\varnothing \text { if } \widetilde{P}_{i} \notin \tilde{p}_{j}
$$

If a joined pair contains a major space, instead of a major point, we define analogously:

$$
\begin{aligned}
& \left(\tilde{S}^{i} \backslash \widetilde{P}_{i}\right) \bowtie \tilde{p}_{j}=\tilde{p}_{j} \text { if } \tilde{p}_{j} \subset\left(\tilde{S}^{i} \backslash \widetilde{P}_{i}\right) ;\left(\tilde{S}^{i} \backslash \widetilde{P}_{i}\right) \bowtie \tilde{p}_{j}=\varnothing \text { if } \tilde{p}_{j} \not \subset\left(\tilde{S}^{i} \backslash \tilde{P}_{i}\right) ; \\
& \left(\tilde{S}^{i} \backslash \tilde{P}_{i}\right) \bowtie \tilde{S}^{j}=\tilde{s}^{j} \text { if } \tilde{s}^{j} \subset\left(\tilde{S}^{i} \backslash \tilde{P}_{i}\right) ;\left(\tilde{S}^{i} \backslash \widetilde{P}_{i}\right) \sim_{\triangleright} \tilde{S}^{j}=\varnothing \text { if } \tilde{s}^{j} \not \subset\left(\tilde{S}^{i} \backslash \tilde{P}_{i}\right)
\end{aligned}
$$

Self-pairs are consequently defined as:

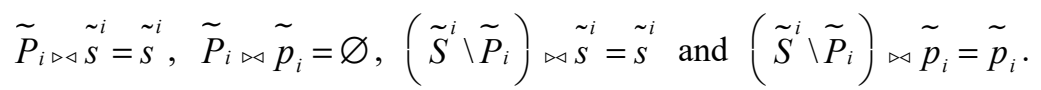

We notice that according to the definitions above, the result of joining in all cases is a minor object. Thus the transformation of a joined pair is equal to the transformation of a minor attribute, similar to the general definition after Equation (3). Having defined joined pairs now, we need to link them, to be able to describe observations. Mind that we will replace the linking of single attributes, as we did in Equation (3), by linking of joined pairs. If we call two major attributes $\mathrm{A}$ and $\mathrm{B}$ and two minor ones $\mathrm{c}$ and $\mathrm{d}$, we can combine them to two joined pairs $A \bowtie b$ and $C_{\triangleright \triangleleft d} d$. Then linking of two joined pairs can be written as $(A \triangleright \triangleleft) \propto\left(C_{\triangleright \triangleleft} d\right)$ and defined as their intersection. Linking is not distributive over joining. Now we are ready to define the transformation of linked joined pairs into a physical space.

The transformation of minor space $\tilde{\sim}^{i}$ is $\left[\widetilde{s^{i}}\right]=s^{i}$, with $s^{i}$ being a small physical space. For the transformation of pellicle $\widetilde{p}_{i}$, which is $\left[\widetilde{p}_{i}\right]$, we need a new definition, because this is a non-singular space object. We define $\left[\widetilde{p}_{i}\right]$ as the transformation of an infinitesimal part of $\widetilde{p}_{i}$ and call it pellicle point $\pi\left(p_{i}\right)$, being a tiny sphere inside the coinciding pellicles, having an extensiveness as large as its width ( $d \boldsymbol{r})$ and touching both borders. The round brackets indicate that the pellicle itself is actually existing as a skin around the minor space, but only the part $\pi\left(p_{i}\right)$ of it is observable. A daily life object like the pellicle point can be found in a bicycle: a ball in a ball bearing. The resulting items, $s^{i}$ and $\pi\left(p_{i}\right)$, are physical observations. We call them small-scale observations.

\subsection{Space Attributes of Coinciding H-units}

Returning to the example of coinciding H-units and using the extended set of attributes (6) together with definitions (7) through (10), we find the joined pairs; in this case all of them reduce to two self-pairs. After transformation we get two small-scale physical observations, which are collected in the set $\mathbf{o}(\mathbf{x})$, called the set of small-scale observations, written as

$$
o(\mathbf{x})=\left\{o_{1}, o_{2}\right\}=\left\{\left[\tilde{s}^{i}\right],\left[\tilde{p}_{i}\right]\right\}=\{s, \pi(p)\} .
$$

The first element can be considered as a spherical mass with the centre in $P$, which is what we wanted to obtain. The second element is a much smaller spherical mass, existing in the pellicle $p$. In contrast with (11), the observations in (5) are called large-scale observations and the set $O(\mathbf{x})$ is called the large-scale set:

$$
O(\mathbf{x})=\left\{O_{1}, O_{2}\right\}=\{P, S \backslash P\}
$$

If we compare (11) with (12), they are very different, (11) containing only small-scale observations and (12) only large-scale observations. Clearly the addition of minor attributes to the interacting H-units leads to a dramatically different result. Concerning the energy, both elements of (11) contain actual energy, whilst one 
element of (12), major point $P$, contains no energy at all. It might seem as if we have to choose between large and small-scale observations, but the world is not split up in large and small events; each event combines both, so we have to reconcile these two types of observations. This is realized by combining each element of the large-scale set with the corresponding element of the small-scale set, thus constituting a subset of two elements. Then (11) and (12) can be combined to one expression, called a space zipper as:

$$
Z\left(h_{1}(\tilde{\mathbf{x}}) * h_{2}(\tilde{\mathbf{x}})\right)=\left\{\left\{O_{1}, o_{1}\right\},\left\{O_{2}, o_{2}\right\}\right\}=\{\{P, s\},\{S \backslash P, \pi(p)\}\} .
$$

This set is the set of all possible observations, large-scale as well as small-scale, of two interacting coinciding H-units having space attributes. Each element of the zipper, being a subset, is called a $\boldsymbol{z} \boldsymbol{i p}$. It describes a physical event which we call a Heisenberg event (shortened to H-event). The two zips of zipper (13) are

$$
z_{1}(\mathbf{x})=\{P, s\} \text { and } z_{2}(\mathbf{x})=\{S \backslash P, \pi(p)\},
$$

so (13) may also be written as

$$
Z\left(h_{1}(\tilde{\mathbf{x}}) * h_{2}(\tilde{\mathbf{x}})\right)=\left\{z_{1}(\mathbf{x}), z_{2}(\mathbf{x})\right\} .
$$

This zipper represents all observational information about the two coinciding, interacting H-units, showing how potential energy is transformed into actual energy in a complementary way. In each zip the large-scale observation is enriched with the corresponding small-scale observation, describing an H-event. Each H-event contains an amount of energy which (in this coinciding example) must be equal to the total potential energy of the two H-units. Consequently each zip describes the same amount of space energy. This can be stored in two distinct ways: in a contracted or an expanded way (Backerra, 2014). If it is stored in a compact way, having a high energy density, the H-event is called a mass; if the space energy is stored in an extended way, having a low density, the H-event is called a space. We assume that each of these two energy densities is a constant.

Zip $z_{1}(\mathbf{x})$ in (14) describes a large-scale physical point $P$ in the centre of a small-scale physical space $s$. Point $P$ cannot store space energy, so all actual energy is stored in minor space $s$ and its energy density must be high. Thus $z_{1}(\mathbf{x})$ describes a mass, called a major particle. Zip $z_{2}(\mathbf{x})$ in (14) describes a large-scale physical space $S \backslash P$ and a small-scale pellicle point $\pi(p)$ at the surface of the major particle, so the energy of $z_{2}(\mathbf{x})$ is divided over two objects. Space $S \backslash P$ is called a major space, storing energy with a low density. Pellicle point $\pi(p)$, existing at the surface of the major particle, is supposed to store the energy with a high density, so it is called a minor particle.

Summarizing, all energy of two coinciding interacting H-units is transformed from potential to actual energy and manifests itself in two complementary H-events. The first is a major particle, having a localized central point, the second is a major space and a minor particle upon the major particle. We are more familiar with the first H-event, the mass, being determinate (the "certain" point) with a relatively small extension (the "uncertainty" of this point). The second $\mathrm{H}$-event is less easy to grasp. The major space is indeterminate (the "uncertain" space) with a relatively small determinate detail, which is the minor particle (the 'certainty' of the space).

Historically space is considered in two distinct ways. On one hand, in the science of the last few ages, space was considered as a huge collection of separate points, each being determinate and thus without energetic content. On the other hand, from the antique time until the end of the 19th century, space was considered as en energetic object called ether (Michelson \& Morley, 1887). Our notion of space as a large, energy carrying space is similar to the idea of ether, but there are two essential differences. The first is that our major space by definition is not infinite but limited. The second is that in our theory, space is not composed of determinate spatial points, but of indeterminate spatial points. Thus each point is conceived as stretched out over the entire space, having the exciting consequence that any influence upon a space will exist instantaneously everywhere.

\section{Time Attributes}

We continue our theoretical construction of a physical object by adding time attributes to the H-unit. Einstein (1916) was convinced that time had to be considered in exactly the same way as space and, inspired by the results of Minkowski (Corry, 1998), carried this out by considering time as the fourth dimension after three spatial dimensions. On the other hand he recommended to connect physical concepts to associated sensory perception, which is not in agreement with the concept of four-dimensional spacetime; nobody experiences space and time as a four-dimensional continuum. Another objection to consider space and time as a four-dimensional continuum is, that it implies that the past is observable, which is in contradiction with any physical experiment. 
If the past would play a role in physics, it should be observable and if it was observable, this would be in the present or in the future. For that reason the past is absent in twin physics.

Our approach is to give time the same mathematical attributes as space, as Einstein suggested, but, differently to his conviction, we consider time as an independent, one-dimensional quality. This is one step further than we did in a previous paper (Backerra, 2012), where time provisionally was considered to have place zero in a four-dimensional time-space. We do define mathematical time attributes identically to each of the space attributes $\widetilde{P}_{i}, \widetilde{S}_{\widetilde{f}^{i}} \widetilde{P}_{i}, \widetilde{p}_{i}$ and $\tilde{s}^{i}$, reduced to one dimension. The four time attributes are indicated as $\widetilde{T}_{i}$, $\widetilde{F}^{i} \backslash \widetilde{T}_{i}, \tilde{\tau}_{i}$ and $\widetilde{f}^{i}$, existing on a finite line segment $\widetilde{F}^{i}$. Thus an H-unit $H_{i}$ is provided with the set of time attributes

$$
h_{i}(\tilde{t})=\left\{\widetilde{T}_{i}, \widetilde{F}^{i} \backslash \widetilde{T}_{i}, \tilde{\tau}_{i}, \widetilde{f}^{i}\right\},
$$

Bear in mind that time attributes are potential items; they only can become actual if H-units interact with each other. First we consider the two major attributes. The first one is indicated by $\widetilde{T}_{i}$ and called the major point of time, which is the first point of the line segment. The remaining part of the line segment, indicated by $\widetilde{F}^{i} \backslash \widetilde{T}_{i}$, is called the future. The interval, being arbitrarily large, is called the "span" (previously (Backerra, 2014) it was called "full time"), with

$$
\widetilde{F}^{i}=\left\{\tilde{t} \mid \widetilde{T}_{i} \leq \tilde{t}<\widetilde{T}_{e i}\right\},
$$

in which $\widetilde{T}_{e i}$ is the end of the interval, excluded from $\widetilde{F}^{i}$. The two minor attributes are $\widetilde{f}^{i}$ and $\tilde{\tau}_{i}$. The flying time $\widetilde{f}^{i}$ is an indeterminate minor attribute, adding uncertainty at a small scale and thus giving a key quality to the set: it provides the set with uncertainty as an addition to the classical conception of time. It is defined by

$$
\widetilde{f}^{i}=\left\{\tilde{t} \mid \widetilde{T}_{i} \leq \tilde{t}<\tilde{t}_{i}\right\}
$$

and conceived as a small interval (in comparison to $\widetilde{F}^{i}$ ), consisting of $\widetilde{T}_{i}$ and an immediately following part of $\widetilde{F}^{i} \backslash \widetilde{T}_{i}$. The flash of time $\tilde{\tau}_{i}$ is is a determinate minor attribute, an infinitesimal small line segment, located at the upper border of $\widetilde{f}^{i}$ and having a width of $d \tilde{t}$, defined by

$$
\tilde{\tau}_{i}=\left\{\tilde{t} \mid \tilde{t}_{i} \leq \tilde{t} \leq \tilde{t}_{i}+d \tilde{t}_{i}\right\}
$$

It is considered as a mathematical "change" of the first order; previously we called it the "minor point of time". By choosing these time attributes, we anchored uncertainty in the basics of time, which has been overlooked in physics until now.

The operations joining, linking and transforming are defined similarly as for space attributes.

Joined pairs of time attributes are, similar to (7) through (10), defined for pairs containing a major point of time as:

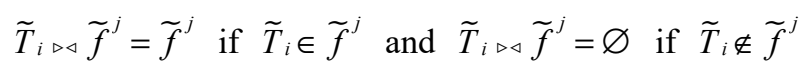

and similar for $\widetilde{T}_{i \triangleright \triangleleft} \tilde{\tau}_{j}$. Joined pairs containing a future are defined as:

$$
\left(\widetilde{F}^{i} \backslash \tilde{T}_{i}\right) \bowtie \tilde{\tau}_{j}=\tilde{\tau}_{j} \text { if } \tilde{\tau}_{j} \subset\left(\widetilde{F}^{i} \backslash \widetilde{T}_{i}\right) \text { and }\left(\widetilde{F}^{i} \backslash \widetilde{T}_{i}\right) \bowtie \tilde{\tau}_{j}=\varnothing \text { if } \tilde{\tau}_{j} \not \subset\left(\widetilde{F}^{i} \backslash \widetilde{T}_{i}\right),
$$

and similar for $\left(\widetilde{F}^{i} \backslash \tilde{T}_{i}\right) \bowtie \widetilde{f}_{j}$. Obviously the self-pairs are defined as

$$
\widetilde{T}_{i \triangleright \triangleleft} \widetilde{f}^{i}=\widetilde{f}^{i} ; \quad \widetilde{T}_{i \triangleright \triangleleft} \tilde{\tau}_{i}=\varnothing ;\left(\widetilde{F}^{i} \backslash \widetilde{T}_{i}\right) \bowtie \widetilde{f}^{i}=\widetilde{f}^{i} \text { and }\left(\widetilde{F}^{i} \backslash \tilde{T}_{i}\right) \bowtie \tilde{\tau}_{i}=\tilde{\tau}_{i}
$$

Linking of joined pairs is defined as their intersection.

The transformation of time attributes into physical observations is defined as an operation which changes a one-dimensional mathematical object into physical time; then the mathematical axis of $\tilde{t}$ transforms into a real time axis $t$. Different as with space attributes, we do not consider time as an energetic object, so we do not 
ascribe energy to transformed time attributes. As a consequence, we suppose that the sizes of time attributes are equal for all H-units.

The transformations are defined by $\left[\widetilde{T}_{i}\right]=T_{i},\left[\widetilde{F}^{i} \backslash \widetilde{T}_{i}\right]=F^{i} \backslash T_{i},\left[\tilde{\tau}_{i}\right]=\tau_{i}$ and $\left[\widetilde{f}^{i}\right]=f^{i}$, in which $T_{i}$ is a physical point of time and the remaining three items are physical time intervals. We will try to connect them to daily life experiences. Major point of time $T_{i}$ can be recognized as the indication of a stopwatch; it may be called the "mathematical presence". Flying time $f^{i}$ is an indeterminate extension of "now"; thus the present is not only conceived as a single point $T_{i}$ upon the time axis, but as the interval $T_{i} \cup f^{i}$, which may be called the "physical presence". This reflects the experience that time is already gone when you press the stopwatch or say "now". Also it reflects the well known experience that any physical measurement needs an interval of time, whatever small it may be. It is also experienced by musicians of an orchestra, playing music and in the same time (the flying time) reading and hearing the notes of the next bar. This is not merely a feeling but a controllable feature of the human brain, as explained by Y. Joosten (Joosten, 2011). In normal situations the brain gathers all incoming information during about 3 seconds, with intervals of 20 tot 40 milliseconds, without distinguishing in time. Then a decision is taken about the 'present situation', after which the brain starts again collecting information. Thus the upper border of the flying time is the right position for the flash of time $\tau_{i}$, describing change. We suppose that this decribes an energetic change, like the acceleration of a particle, gaining kinetic energy. The future $F^{i} \backslash T_{i}$ is all remaining time of existence after the mathematical presence. The part of the future after $T_{i} \cup f^{i}$ may be called the "physical future". If two H-units are interacting, the time attributes become actual; however, depending on the type of interaction, interaction does not imply that time will run self-evident. Time runs only if a cyclic phenomenon is generated by the interaction, providing a clock. If the interaction is such that time indeed is running, a one-dimensional time axis will be generated and $T_{i}$ moves along this axis, taking the remaining attributes with it.

\subsection{Time Attributes of Coinciding H-Units}

For the example of coinciding H-units, the time zipper is, analogous to (13), the set with two elements

$$
Z(t)=\left\{z_{1}(t), z_{2}(t)\right\}=\{\{T, f\},\{F \backslash T, \tau\}\},
$$

in which a one-dimensional infinitesimal interval is analogous to the pellicle point. According to the large-scale observations at the left side of each zip, $z_{1}(t)$ is observed in the major point of time $T$ and $z_{2}(t)$ in the future $F \backslash T$. According to the small-scale observations at the right side of each zip, $z_{1}(t)$ is observed during the flying time $f$ (including $T$ ) and $z_{1}(t)$ in flash of time $\tau$, immediately after $f$. Combining with space zipper (13), we obtain time-space zipper

$$
Z\left(h_{1}(\tilde{t}, \tilde{\mathbf{x}}) * h_{2}(\tilde{t}, \tilde{\mathbf{x}})\right)=\left\{z_{1}(t, \mathbf{x}), z_{2}(t, \mathbf{x})\right\}=\left\{\begin{array}{l}
\{\{T, P\},\{f, s\}\} \\
\{\{F \backslash T, S \backslash P\},\{\tau, \pi(p)\}\}
\end{array}\right\} .
$$

The large-scale observation of zip $z_{1}(t)$ is $\{T, P\}$, its small-scale observation is $\{f, s\}$. In combination, the $\mathrm{H}$-event is described in the physical presence as a localized, spherical particle, having a small spatial extension. This is a major particle without electromagnetic features, which can be identified as a dark major particle. Because we expressed the interaction in coordinates related to the coinciding H-units itself, there is nothing to move to or from, and thus its velocity is undetermined. The large-scale observation of zip $z_{2}(t)$ is $\{F \backslash T, S \backslash P\}$, its small scale observation is $\{\tau, \pi(p)\}$. In combination, the generated H-event is described in the flash of time $\tau$ as a major space in which pellicle point $\pi(p)$ exists at the surface of the dark major particle as a minor particle.

Because $\tau$ describes a change, $\pi(p)$ can only be observed if it is changing. If we suppose that this has to be a change in energetic sense, it can only be a change of velocity, so it has to move accelerated over the surface.

This can go on until the sum of its rest mass and its kinetic energy is equal to the rest mass of the major particle, a moment which is supposed to be situated at the upper border of $\tau$. After that, the particle cannot increase its energy anymore because it cannot have more energy than the major particle. Because then there is no time element available to describe this, $\pi(p)$ is not observable anymore, although it continues to exist as $F \backslash T$ is described in the large-scale part of the zip. In the future after $\tau$, the particle exists independent of time, so it will have a constant velocity. In our second paper (Backerra, 2012) we identified $\pi(p)$ as minor dark particles in several cases, which are different from this one because both H-units were marked and a neutral $\mathrm{H}$-unit was involved as a background. There we supposed that, after escaping from the surface, this particle can 
be identified as a neutrino, a particle with a tiny mass, having a constant velocity very close to the velocity of light. For that reason $\pi(p)$ in this case may be considered as an embryonic neutrino. We will return to this example after having considered zippers in general and having derived the attributes of mark, to involve electromagnetic features.

The considerations above show that a time observation acts as a label, attached to a spatial observation, informing us about the status of the energy and so about the type of existence, potential or actual. An empty label indicates a potential physical existence, being timeless; a non-empty label indicates the actual existence of an H-event, even it is not observable, and informs us whether it occurs in major point of time $T$, flying time $f$ or future $F \backslash T$, and whether it is moving with a constant velocity (indicated by $T$ or $f$ ) or accelerated (indicated by $\tau$ ). Because the first thing you want to know about an event is, whether it is potential or actual, we placed time before space in the zipper.

\section{The Zipper in General}

The transformation of joined and linked pairs of time and space of interacting $\mathrm{H}$-units $H_{i}$ and $H_{j}$ into physical observations is in general a time-space zipper which analogous to (13) can be written as the set with $\mathrm{n}$ elements

$$
Z\left(h_{i}(\tilde{t}, \tilde{\mathbf{x}}) * h_{j}(\tilde{t}, \tilde{\mathbf{x}})\right)=\left\{\left\{O_{1}(t, \mathbf{x}), o_{1}(t, \mathbf{x})\right\},\left\{O_{2}(t, \mathbf{x}), o_{2}(t, \mathbf{x})\right\}, \ldots,\left\{O_{n}(t, \mathbf{x}), o_{n}(t, \mathbf{x})\right\}\right\} .
$$

One element of the zipper is a zip $z_{n}(t, \mathbf{x})$, which in general, analogous to (14), can be written as the set of two elements

$$
z_{n}(t, \mathbf{x})=\left\{O_{n}(t, \mathbf{x}), o_{n}(t, \mathbf{x})\right\},
$$

in which $O_{n}(t, \mathbf{x})$ and $o_{n}(t, \mathbf{x})$ are, respectively, the transformation of a large- and a small-scale observation. Analogous to (15), the zipper may also be written as

$$
Z\left(h_{i}(\tilde{t}, \tilde{\mathbf{x}}) * h_{j}(\tilde{t}, \tilde{\mathbf{x}})\right)=\left\{z_{1}(t, \mathbf{x}), z_{2}(t, \mathbf{x}), \ldots, z_{n}(t, \mathbf{x})\right\} .
$$

The $\mathrm{n}$ zips can be derived for specific cases by using the set of time attributes (16)

$$
h_{i}(\tilde{t})=\left\{\widetilde{T}_{i}, \widetilde{F}^{i} \backslash \widetilde{T}_{i}, \tilde{\tau}_{i}, \widetilde{f}^{i}\right\}
$$

and the set of space-attributes (6)

$$
h_{i}(\tilde{\mathbf{x}})=\left\{\widetilde{P}_{i}, \widetilde{S}^{i} \backslash \widetilde{P}_{i}, \tilde{p}_{i}, \tilde{s}^{i}\right\} .
$$

It might be practical to write the general set of time-space attributes for one $\mathrm{H}$-unit $H_{i}$ as a combination of (28) and (29), so as

$$
h_{i}(\tilde{t}, \tilde{\mathbf{x}})=\left\{\left\{\widetilde{T}_{i}, \widetilde{P}_{i}\right\},\left\{\widetilde{F}^{i} \backslash \widetilde{T}_{i}, \widetilde{S}^{i} \backslash \widetilde{P}_{i}\right\},\left\{\tilde{\tau}_{i}, \widetilde{p}_{i}\right\},\left\{\widetilde{f}^{i}, \tilde{s}^{i}\right\}\right\} .
$$

In the example of coinciding $\mathrm{H}$-units it was possible to derive the joined pairs, links and transformations without a general formulation, but in other cases this will be not so easy. In general H-units are not coinciding, not in geometric, nor in time attributes, so we need to find a general expression for the zips in any specific case, expressed in physical items, being the H-events. For convenience we repeat the general set of attributes $h_{i}$ of H-unit $H_{i}$ (see (1)):

$$
h_{i}=\left\{D_{i}, U^{i}, d_{i}, u^{i}\right\}
$$

As we showed in section 3 (above Equation (7)), two H-units produce maximum 16 joined pairs, including self-pairs, so when they are linked to chains, a chain can contain maximum 16 distinct joined pairs, instead of only 2 in time-space zipper (24). This can be reduced by remembering that quantum mechanics tells us that complementary observations cannot be observed simultaneously. This can be used to formulate a restriction, called the exclusion principle, saying that a joined pair, containing a determinate major attribute, cannot link with a joined pair, containing an indeterminate major attribute of the same H-unit and the same quality. An 
example of a forbidden link is $\left(D_{1} \bowtie u^{1}\right) \propto\left(U^{1} \bowtie u^{2}\right) ;$ an example of an allowed link is $\left(D_{2} \bowtie u^{1}\right) \propto\left(U^{1} \triangleright u^{2}\right)$

A consequence of the exclusion principle is, that in general chains of maximum four joined pairs can be transformed into H-events. Because sets with chains of three joined pairs didn't add more information, we used only chains of two or four joined pairs. Using chains of two joined pairs, the zipper of the first order can be derived, $Z^{1}$, containing eight zips. Using chains of four joined pairs, the zipper of the second order can be derived, $Z^{2}$, containing four zips.

All together there exist twelve distinct chains, each adding a small scale observation to a corresponding large-scale observation, producing 12 distinct zips. Another consequence of the exclusion principle is, that distinct zips of a specific interaction cannot be observed simultaneously.

In dozens of specific cases, only two zips of a zipper turned out to be non-empty, in $Z^{1}$ as well as in $Z^{2}$, and thus can be transformed to physical events. In this paper we use only zippers of the second order, because with the first order zipper we found no other results than gravitation (Backerra, 2014), whilst in this paper we are searching for the photon.

According to the derivation in our second paper (Backerra, 2012), the zipper of the second order for two interacting H-units $H_{i}$ and $H_{j}$, having sets of attributes $h_{i}$ and $h_{j}$, can in general be written as the set of four elements

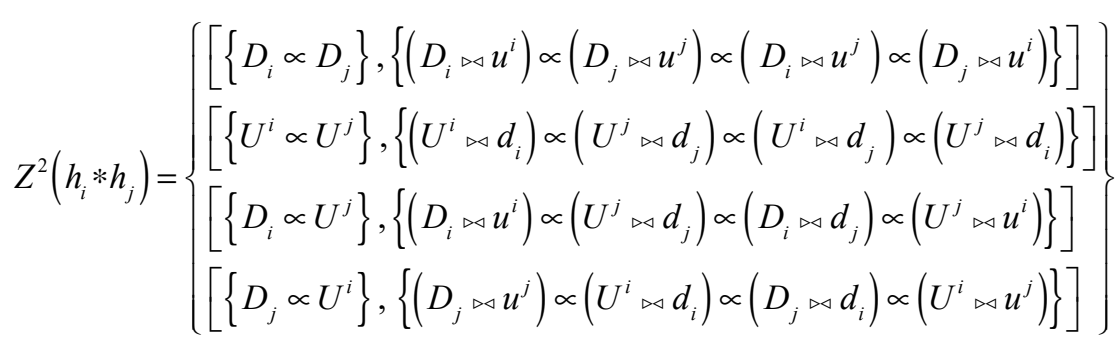

As a verification, we insert the attributes of coinciding $\mathrm{H}$-units in (32), so

$$
D_{i}=D_{j}=\{\widetilde{P}, \widetilde{T}\}, d_{i}=d_{j}=\{\tilde{p}, \tilde{\tau}\}, U^{i}=U^{j}=\{\widetilde{S} \backslash \widetilde{P}, \widetilde{F}\} \text { and } u^{i}=u^{j}=\{\tilde{s}, \widetilde{f}\},
$$

carry out joining and linking. After transforming them, indeed we obtain time-space zipper (24).

The index 2 of the zipper, indicating second order, will be dropped in the following. Each element of the zipper is a zip, containing an amount of potential or actual energy which is equal for all zips of a specific zipper, depending on the intensity of the interaction. By carrying out the transformation from mathematics to physics, the tildes above the attributes and the square brackets disappear. Then each zip contains physical items, describing one H-event. Because of the principle of exclusion, distinct zips cannot be observed simultaneously, so when observing one zip, the other zips are not observable, although they may contain actual energy. Until now, in each case at the most two H-events appear. The large-scale element of a zip (at the left) contains two major attributes, linked to each other; after applying linking and transforming, this element reduces to a large-scale observation. The small-scale element of a zip (at the right) contains four joined pairs, linked to each other; after applying the operations joining, linking and transforming, this element reduces to a small scale observation.

In this way a zipper acts as a bridge between large- and small-scale physics. Requiring that corresponding large and small scale observations are compatible, zips are only observable if large and small observations have at least one point in common. If not, the complete zip is replaced by an empty set and its energy stays potential. This requirement can be compared with a closed every-day zipper, having left and right "teeth" which have to be in contact with each other to be useful.

Inserting the general time attributes (16) in (32), we obtain the general time zipper of the second order as 


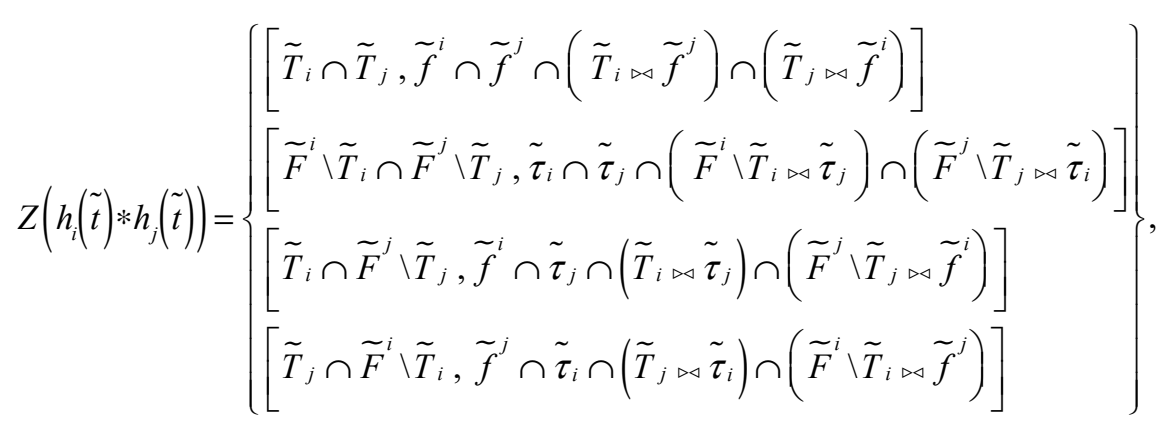

The time zipper is completely defined by the positions of $\widetilde{T}_{i}$ and $\widetilde{T}_{j}$ with respect to each other.

Inserting the general space attributes (6) in (32), we obtain the general space zipper of the second order as

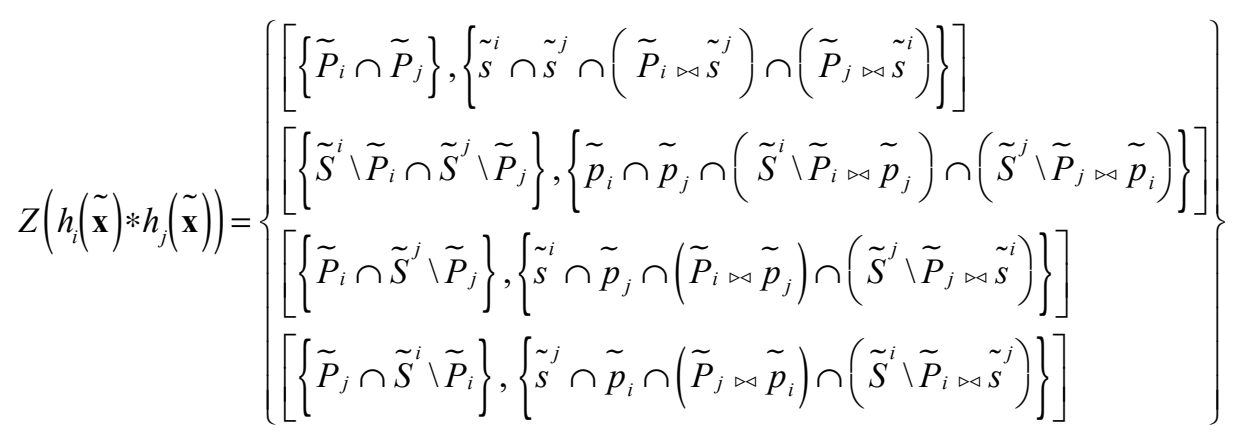

The space zipper is completely defined by the positions of $\widetilde{P}_{i}$ and $\widetilde{P}_{j}$ with respect to each other.

Any time-space interaction can be characterized by a time case together with a space case and in principle they may be distinct. However, in this paper, following the route of Einstein as suggested by Minkowski to treat space and time in the same way, we will restrict ourselves to identical time and space cases.

In the following, $Z\left(h_{i}(\tilde{t}, \tilde{\mathbf{x}}) * h_{j}(\tilde{t}, \tilde{\mathbf{x}})\right)$ will be indicated as $Z_{i j}(t, \mathbf{x})$. This is not only more convenient, but also expresses the physical character of the zipper, after having carried out the transformation. Consequently, the zipper of time will be written as $Z_{i j}(t)$ and the zipper of space as $Z_{i j}(\mathbf{x})$. The zipper can be reduced in specific time and space cases and transformed to descriptions of actual phenomena. For convenience we repeat: Time transformations are defined by $\left[\widetilde{T}_{i}\right]=T_{i} \widetilde{T}^{2}\left[\widetilde{F}^{i} \backslash \widetilde{T}_{i}\right]=F^{i} \backslash T_{i},\left[\tilde{\tau}_{i}\right]=\tau_{i}$ and $\left[\widetilde{f}^{i}\right]=f^{i} ;$ space transformations are defined by $\left.[\widetilde{P}]=P,[\widetilde{S} \backslash \widetilde{P}]=S \backslash P, \widetilde{S^{i}}\right]=s^{i}$ and $\left[p_{i}\right]=\pi\left(p_{i}\right)$. If a zip is empty, so $z_{i}=\varnothing$, than it describes potential energy. If the transformation of a small-scale element is empty in a certain quality, the large-scale element of the same zip is not observable, as a consequence of the extended Heisenberg's uncertainty principle, although it may describe actual energy. Mind that previously unobservable items were removed from the zipper, which was adequate for the considered cases in which we were searching for descriptions of mass. In that instance we described "potential items" in mathematical terms and "observed items" in physical terms. In this paper we discovered that actual energetic items may be described without being observable. For that reason we changed the term "observed items" in "actual items" and split them in two possibilities, called "observable actual items and "unobservable actual items". Unobservable items will be placed between round brackets.

\section{Mark Attributes}

In order to describe photons, we have to introduce mathematical attributes which, after interaction with another H-unit and transforming into physical observations, can describe electromagnetic fields. These attributes are called marks. This may appear to be a difficult part of the theory, but in fact it is rather straightforward, without requiring complicated mathematical operations. Mark attributes have to be defined in a complementary way, analogous to what we did with space and time attributes, and such that an entire $\mathrm{H}$-unit is distinguished from another one as if they were painted 'red' and 'blue'. Moreover, if the H-unit is not marked, it has to be 'colourless'. We start the derivation by realizing that the entire potential presence of an H-unit is its major space $\widetilde{S}^{i}$, existing in major point of time $\widetilde{T}_{i}$ and future $\widetilde{F}^{i} \backslash \widetilde{T}_{i}$. The spherical shape of $\widetilde{S}^{i}$ makes it suitable to be marked by vector fields.

For the major determinate mark attribute we choose a 3-dimensional, radial oriented, time dependent vector field $\widetilde{\mathbf{E}}_{i}(\tilde{t})$, having a source of strength $\widetilde{Q}_{i}$ at point $\widetilde{P}_{i}$. The tildes indicate that these are mathematical items, 
which later will be transformed into a physical items. $\widetilde{\mathbf{E}}_{i}(\tilde{t})$ is called the determinate field; for convenience its mathematical time dependency will be suppressed. Applying the divergence theorem of the first law of Gauss yields the requirement

$$
\nabla \cdot \widetilde{\mathbf{E}}_{i}=\widetilde{Q}_{i}
$$

One vector of $\tilde{\mathbf{E}}_{i}$, indicated by $\tilde{\mathbf{e}}_{i}$, is called positive if it points from $\widetilde{P}_{i}$ to infinity. In $\widetilde{P}_{i}$ the field is defined as the zero vector $\mathbf{0}$.

For the major indeterminate mark attribute we choose a 3-dimensional time dependent vector field $\widetilde{\mathbf{B}}^{i}(\tilde{t})$ with central point $\widetilde{P}_{i}$, having one vector in each point. This is called the indeterminate field; its mathematical time dependency will be suppressed. A vector of $\widetilde{\mathbf{B}}^{i}$, indicated by $\widetilde{\mathbf{b}}^{i}$, has a direction tangent to a spherical surface with $\widetilde{P}_{i}$ as the central point; adjacent vectors may point in an infinite variety of directions and moreover there is no information about the absolute value of $\tilde{\mathbf{b}}^{p}$. In $\widetilde{P}_{i}$ the field is defined as the zero vector o. To be absolutely sure that it is an indeterminate vector field, we add the principle of uniqueness to the definition of $\widetilde{\mathbf{B}}^{i}$, which $\widetilde{\mathbf{B}}_{i}$ says that $\widetilde{\mathbf{B}}^{i} \neq \widetilde{\mathbf{B}}^{j}$ for any two H-units $H_{i}$ and $H_{j}$. With these two definitions, the marking-fields $\widetilde{\mathbf{E}}_{i}$ and $\widetilde{\mathbf{B}}^{i}$ are in each point perpendicular to each other, so $\widetilde{\mathbf{E}}_{i} \cdot \widetilde{\mathbf{B}}^{i}=\mathbf{O}$ and, because $\widetilde{\mathbf{E}}_{i}$ is radial,

$$
\nabla \cdot \widetilde{\mathbf{B}}^{i}=0
$$

We are not ready yet, because in $\widetilde{P}_{i}$ both fields are defined as zero, so this point of $\widetilde{S}^{i}$ is not marked complementary. In order to mark $\widetilde{P}_{i}$ in a determinate way, a real number $\widetilde{Q}_{i}$ is attached to it. To mark $\widetilde{P}_{i}$ in an indeterminate way, an imaginary number $\widetilde{Q}_{i} \times i$ (with $i=\sqrt{-1}$ ) is attached to it. Then the total major determinate mark of $H_{i}$ can be written as the set with two elements $\left\{\widetilde{\mathbf{E}}_{i}, \widetilde{Q}_{i}\right\}$ and the total major indeterminate one as the set with two elements $\left\{\widetilde{\mathbf{B}}^{i}, \widetilde{Q}_{i} \times i\right\}$.

Next we need minor attributes of mark. They may be chosen in an arbitrary way, as long as they are complementary and exclude each other. We choose two operators having a connection with the fields, such that joined pairs can get a vectorial character in space and time. The minor determinate mark attribute for the vector fields of H-unit $H_{i}$ is chosen as $\widetilde{\nabla}$, with $\widetilde{\nabla}=\partial / \partial \tilde{x}, \partial / \partial \tilde{y}, \partial / \partial \tilde{z}$, and the minor indeterminate mark attribute as $\partial / \partial \tilde{t}$. The absence of indices indicates that these attributes are identical for all marked H-units. They exclude each other and together constitute an infinitesimal unit of space and time. Again we are not ready yet before having marked als $\widetilde{P}_{i}$ in a complementary way. We define the minor determinate mark attribute for $\widetilde{P}_{i}$ as the real number 1 and the minor indeterminate mark attribute as the imaginary number $i$ (with $i=\sqrt{-1}$ ). Than the set of mark attributes of H-unit $H_{i}$ can be written as

$$
h_{i}(\tilde{q})=\left\{\left\{\widetilde{\mathbf{E}}_{i}, \widetilde{Q}_{i}\right\},\left\{\widetilde{\mathbf{B}}^{i}, \widetilde{Q}_{i} \times i\right\}, \widetilde{\nabla}, \partial / \partial \tilde{t}\right\},
$$

in which $\tilde{q}$ indicates the quality "mark". We have to define the operations joining, linking and transforming of mark attributes separately for fields (describing the space around $\widetilde{P}_{i}$ ) and numbers (describing only $\widetilde{P}_{i}$ ). Joined pairs for fields are defined by allowing minor attributes to act upon major attributes, such that field variations are introduced in the descriptions. We choose to define joining of vector field $\widetilde{\mathbf{A}}$ with, subsequently, the minor attributes $\widetilde{\nabla}$ and $\partial / \partial \tilde{t}$ by

$$
\widetilde{\mathbf{A}}_{\triangleright} \widetilde{\nabla}=\widetilde{\nabla} \times \widetilde{\mathbf{A}} \text { and } \tilde{\mathbf{A}} \bowtie \partial / \partial \tilde{t}=\partial \tilde{\mathbf{A}} / \partial \tilde{t}
$$

in which $\widetilde{\nabla} \times \widetilde{\mathbf{A}}$ is a vector-product. Two examples of the joined pairs in (32) are

$$
D_{i} \bowtie u^{i}=\tilde{\mathbf{E}}_{i \triangleright \triangleleft} \partial / \partial \tilde{t}=\partial \tilde{\mathbf{E}}_{i} / \partial \tilde{t} \text { and } U^{i} \bowtie d_{i}=\tilde{\mathbf{B}}^{i} \bowtie \widetilde{\nabla}=\widetilde{\nabla} \times \tilde{\mathbf{B}}^{i},
$$

In similar way all joined pairs of $H_{i}$ and $H_{j}$ are obtained as

$$
\partial \widetilde{\mathbf{E}}_{i} / \partial \tilde{t}, \partial \widetilde{\mathbf{E}}_{j} / \partial \tilde{t}, \partial \widetilde{\mathbf{B}}^{i} / \partial \tilde{t}, \partial \widetilde{\mathbf{B}}^{j} / \partial \tilde{t}, \widetilde{\nabla} \times \widetilde{\mathbf{B}}^{i}, \widetilde{\nabla} \times \widetilde{\mathbf{B}}^{j}, \widetilde{\nabla} \times \widetilde{\mathbf{E}}_{i} \text { and } \widetilde{\nabla} \times \widetilde{\mathbf{E}}_{j} .
$$

Because minor mark attributes are identical for all H-units, there is no distinction between mixed pairs and self-pairs. 
Inserting the major mark attributes and the joined pairs (41) in (32), we obtain the general mark zipper of the second order for $H_{i}$ and $H_{j}$ as

$$
Z(q)=\left\{\begin{array}{l}
{\left[\left\{\widetilde{Q}_{i} \propto \widetilde{Q}_{j}, \widetilde{\mathbf{E}}_{i} \propto \widetilde{\mathbf{E}}_{j}\right\}, \partial \widetilde{\mathbf{E}}_{i} / \partial \tilde{t} \propto \partial \widetilde{\mathbf{E}}_{j} / \partial \tilde{t}\right]} \\
{\left[\left\{\left\{\left\{\widetilde{Q}_{i} \times i\right\} \propto\left\{\widetilde{Q}_{j} \times i\right\}, \widetilde{\mathbf{B}}^{i} \propto \widetilde{\mathbf{B}}^{j}\right\}\right\},\left\{\widetilde{\nabla} \times \widetilde{\mathbf{B}}^{i} \propto \widetilde{\nabla} \times \widetilde{\mathbf{B}}^{j}\right\}\right]} \\
{\left[\left\{\widetilde{Q}_{i} \propto\left\{\widetilde{Q}_{j} \times i\right\}, \widetilde{\mathbf{E}}_{i} \propto \widetilde{\mathbf{B}}^{j}\right\}, \partial \widetilde{\mathbf{E}}_{i} / \partial \tilde{t} \propto \widetilde{\nabla} \times \widetilde{\mathbf{B}}^{j} \propto \widetilde{\nabla} \times \widetilde{\mathbf{E}}_{i} \propto \partial \widetilde{\mathbf{B}}^{j} / \partial \tilde{t}\right]} \\
{\left[\left\{\widetilde{Q}_{j} \propto\left\{\widetilde{Q}_{i} \times i\right\}, \widetilde{\mathbf{E}}_{j} \propto \widetilde{\mathbf{B}}^{i}\right\}, \partial \widetilde{\mathbf{E}}_{j} / \partial \tilde{t} \propto \widetilde{\nabla} \times \widetilde{\mathbf{B}}^{i} \propto \widetilde{\nabla} \times \widetilde{\mathbf{E}}_{j} \propto \partial \widetilde{\mathbf{B}}^{i} / \partial \tilde{t}\right]}
\end{array}\right\} .
$$

Mind that the operation linking $(\propto)$ is carried out after the operation joining (involving $\partial / \partial \tilde{t}$ and $\widetilde{\nabla}$ ). This set looks awfully complicated, but after having deduced limiting conditions, in specific cases the set can be reduced to a more manageable device.

Linking of joined pairs for fields is defined as vector addition. Bear in mind that, according to the definition, linking of equal vector fields results in a single vector field, so if $\widetilde{\mathbf{E}}_{i}=\widetilde{\mathbf{E}}_{j}$ than $\widetilde{\mathbf{E}}_{i} \propto \widetilde{\mathbf{E}}_{j}=\widetilde{\mathbf{E}}_{i}$.

Transformation for fields is defined as an operation which changes mathematical fields in electromagnetic fields and ascribes electromagnetic energy to it. The transformations are defined by:

$$
\left[\widetilde{\mathbf{E}}_{i}\right]=\mathbf{E}_{i} ;\left[\widetilde{\mathbf{B}}^{i}\right]=\mathbf{B}^{i} ;\left[\widetilde{\nabla} \times \widetilde{\mathbf{E}}_{i}\right]=\nabla \times \mathbf{E}_{i} ;\left[\widetilde{\nabla} \times \widetilde{\mathbf{B}}^{i}\right]=\nabla \times \mathbf{B}^{i}
$$

and so on, with $\mathbf{E}_{i}$ being a time-dependent electric field, $\mathbf{B}^{i}$ being a time-dependent magnetic field, in which the dependency of physical time $t$ is suppressed. A zero field transforms into an empty set, so $[\mathbf{O}]=\varnothing$.

Joined pairs for numbers are defined by multiplication of major attributes with, subsequently, minor attributes 1 and $\mathrm{i}$ : so

$$
\widetilde{Q}_{i} \times 1, \quad \widetilde{Q}_{i} \times i, \quad \widetilde{Q}_{i} \times i,\left(\widetilde{Q}_{i} \times i\right) \times i, \quad \widetilde{Q}_{j} \times 1, \widetilde{Q}_{j} \times i, \quad\left(\widetilde{Q}_{j} \times i\right) \times 1 \text { and }\left(\widetilde{Q}_{j} \times i\right) \times i,
$$

which can be reduced to the numbers

$$
\widetilde{Q}_{i}, \widetilde{Q}_{i} \times i,-\widetilde{Q}_{i}, \widetilde{Q}_{j}, \widetilde{Q}_{j} \times i \text { and }-\widetilde{Q}_{j} .
$$

Linking of joined pairs for numbers is defined as numerical addition. By definition, linking of equal numbers results in a single number, so if $\widetilde{Q}_{i}=\widetilde{Q}_{j}$ than $\widetilde{Q}_{i} \propto \widetilde{Q}_{j}=\widetilde{Q}_{i}$.

Transformations of numbers is defined as an operation which changes mathematical attributes in electromagnetic charges and ascribes electromagnetic energy to it, by taking the real part of the complex number. Thus

$$
\left[\widetilde{Q}_{i}\right]=Q_{i},\left[\widetilde{Q}_{i} \times i\right]=0 \text { and }\left[-\widetilde{Q}_{i}\right]=-Q_{i},
$$

in which $Q_{i}$ is a positive electric charge, so interacting marked H-units may produce positive, negative or zero charge.

Before being able to reduce mark zipper (42) for specific cases, we have to introduce limiting conditions. These were derived in our first paper, but that derivation was rather unclear and contained errors. In the next section we present a slightly alternative deduction by considering the mark zipper for two coinciding H-units $H_{1}$ and $H_{2}$, being marked equally.

\subsection{The Mark Zipper of Equally Marked Coinciding H-units}

"Equally marked" means nothing more than that the major determinate marks are equal, so

$$
\widetilde{Q}_{2}=\widetilde{Q}_{1}=\widetilde{Q} \text { and } \widetilde{\mathbf{E}}_{2}=\widetilde{\mathbf{E}}_{1}=\widetilde{\mathbf{E}},
$$

because the minor marks of all $\mathrm{H}$-units are defined as equal and because of Equation (36), even equal numbers are enough to define this case. For major indeterminate marks, equally marking is not possible, due to the principle of uniqueness saying that $\widetilde{\mathbf{B}}^{j} \neq \widetilde{\mathbf{B}}^{i}$ for any two H-units. 
Major determinate mark attributes are marked such, that an entire H-unit is distinguished from another one. In the first part of section 6 we gave the example of painting their major spaces in different colors. Obviously, painting two coinciding H-units in one color has to result in the same color, without mixed tones. Similarly, equal marks in the form of numbers and vector fields are supposed to produce no mixed mathematical items in the resulting zipper. This can be expressed by the requirement that all small scale joined pairs in the mark zipper are empty.

Inserting (47) in (42) for two H-units $H_{1}$ and $H_{2}$, we obtain the mark zipper of equally marked $\mathrm{H}$-units as

$$
Z(q)=\left\{\begin{array}{l}
{[\{\widetilde{Q}, \widetilde{\mathbf{E}}\}, \partial \widetilde{\mathbf{E}} / \partial \tilde{t}]} \\
{\left[\left\{\left\{\widetilde{Q} \times i, \widetilde{\mathbf{B}}^{1} \propto \widetilde{\mathbf{B}}^{2}\right\}\right\},\left\{\widetilde{\nabla} \times \widetilde{\mathbf{B}}^{1} \propto \widetilde{\nabla} \times \widetilde{\mathbf{B}}^{2}\right\}\right]} \\
\left.\left[\left\{\{\widetilde{Q}, \widetilde{\mathbf{E}}\} \propto\left\{\widetilde{Q} \times i, \widetilde{\mathbf{B}}^{2}\right\}\right\}, \partial \widetilde{\mathbf{E}} / \partial \tilde{t} \propto \widetilde{\nabla} \times \widetilde{\mathbf{B}}^{2} \propto \widetilde{\nabla} \times \widetilde{\mathbf{E}} \propto \partial \widetilde{\mathbf{B}}^{2} / \partial \tilde{t}\right]\right] . \\
\left.\left[\left\{\{\widetilde{Q}, \widetilde{\mathbf{E}}\} \propto\left\{\widetilde{Q} \times i, \widetilde{\mathbf{B}}^{1}\right\}\right\}, \partial \widetilde{\mathbf{E}} / \partial \tilde{t} \propto \widetilde{\nabla} \times \widetilde{\mathbf{B}}^{1} \propto \widetilde{\nabla} \times \widetilde{\mathbf{E}} \propto \partial \widetilde{\mathbf{B}}^{1} / \partial \tilde{t}\right]\right]
\end{array}\right.
$$

To obtain limiting conditions, we require that major determinate mark features may become observable as an addition to large-scale observations, but never in small-scale observations, because the last ones are generated by joining of attributes, which are not allowed to produce anything new in the coinciding case. Thus all small scale observations have to be empty. We will consider subsequently each of the four zips of (48), to find out which restrictions are needed to meet this requirement.

In zip $z_{1}(q)$ the joined pairs are reduced to $\partial \widetilde{\mathbf{E}} / \partial \tilde{t}$. If we take a look at time-zipper (23) for the coinciding case, we see that $z_{1}(q)$ does not contain $\tau$, so no changes can be described. Thus, after transforming this element and combining it with the time zipper, it will be considered as not compatible, so later in the derivation $\partial \widetilde{\mathbf{E}} / \partial \tilde{t}$ has to be replaced by an empty set. In zip $z_{2}(q)$ the joined pairs are $\nabla \times \mathbf{B}^{1} \propto \nabla \times \mathbf{B}^{2}$ and because $\widetilde{\mathbf{B}}^{2} \neq \widetilde{\mathbf{B}}^{1}$, they will not disappear in general. A solution can be obtained by requiring that the joined pairs of $z_{2}(q)$ are in general equal to those of $z_{1}(q)$, so

$$
\widetilde{\nabla} \times \widetilde{\mathbf{B}}^{i}=\partial \widetilde{\mathbf{E}}_{i} / \partial \tilde{t}
$$

by which restriction they can be reduced as

$$
\widetilde{\nabla} \times \widetilde{\mathbf{B}}^{1} \propto \widetilde{\nabla} \times \widetilde{\mathbf{B}}^{2}=\partial \widetilde{\mathbf{E}} / \partial \tilde{t} \propto \partial \widetilde{\mathbf{E}} / \partial \tilde{t}=\partial \widetilde{\mathbf{E}} / \partial \tilde{t}
$$

The result is, that after transforming and combining with the time zipper, the joined pairs of $z_{2}(q)$ also will replaced by an empty set. Using condition (49), the joined pairs of zip $z_{3}(q)$ can be reduced to $\partial \widetilde{\mathbf{E}} / \partial \tilde{t} \propto \widetilde{\nabla} \times \widetilde{\mathbf{E}} \propto \partial \widetilde{\mathbf{B}}^{2} / \partial \tilde{t}$. Neglecting $\partial \widetilde{\mathbf{E}} / \partial \tilde{t}$ for reasons as we explained above, the remaining part is $\widetilde{\nabla} \times \widetilde{\mathbf{E}} \propto \partial \widetilde{\mathbf{B}}^{2} / \partial \tilde{t}$. By requiring this to be zero in general, we obtain the second condition:

$$
\widetilde{\nabla} \times \widetilde{\mathbf{E}}=-\partial \widetilde{\mathbf{B}}^{2} / \partial \tilde{t}
$$

reducing the remaining part of $z_{3}(q)$ to zero. Zip $z_{4}(q)$ can be considered similarly.

In our previous deduction of limiting conditions (Backerra, 2010), we obtained not only limiting conditions (49) and (51), but also a third one, requiring that the transformation of $\widetilde{\mathbf{B}}^{i}$ in general is a zero vector field. As seen later, this is erroneous. Applying the two deduced conditions (49) and (51) on mark zipper (48), it reduces to

$$
Z(q)=\left\{\begin{array}{l}
{[[\{\widetilde{Q}, \widetilde{\mathbf{E}}\}, \partial \widetilde{\mathbf{E}} / \partial \tilde{t}]} \\
{\left[\left\{\left\{\widetilde{Q} \times i, \widetilde{\mathbf{B}}^{1} \propto \widetilde{\mathbf{B}}^{2}\right\}\right\}, \partial \widetilde{\mathbf{E}} / \partial \tilde{t}\right]} \\
{\left[\left\{\{\widetilde{Q}, \widetilde{\mathbf{E}}\} \propto\left\{\widetilde{Q} \times i, \widetilde{\mathbf{B}}^{2}\right\}\right\}, \partial \widetilde{\mathbf{E}} / \partial \tilde{t}\right]} \\
{\left[\left\{\{\widetilde{Q}, \widetilde{\mathbf{E}}\} \propto\left\{\widetilde{Q} \times i, \widetilde{\mathbf{B}}^{1}\right\}\right\}, \partial \widetilde{\mathbf{E}} / \partial \tilde{t}\right]}
\end{array} .\right.
$$


Carrying out the transformations, the link-operators $\propto$ change into + and the tildes disappear. The resulting mark zipper for equally marked, coinciding $\mathrm{H}$-units is

$$
Z(q)=\left\{\begin{array}{l}
\{\{Q, \mathbf{E}\}, \partial \mathbf{E} / \partial t\} \\
\left\{\left\{0,\left(\mathbf{B}^{1}+\mathbf{B}^{2}\right)\right\}, \partial \mathbf{E} / \partial t\right\} \\
\left\{\left\{Q, \mathbf{E}+\mathbf{B}^{2}\right\}, \partial \mathbf{E} / \partial t\right\} \\
\left\{\left\{Q, \mathbf{E}+\mathbf{B}^{1}\right\}, \partial \mathbf{E} / \partial t\right\}
\end{array}\right\} .
$$

Now we obtained four zips, expressing electromagnetic fields and charges. It depends on the time-space-case if they are observable, so we combine this zipper with time-space zipper (24), in which the $3 \mathrm{~d}$ and 4 th zips are empty. The resulting time-space-mark zipper is

$$
Z(t, \mathbf{x}, q)=\left\{\begin{array}{l}
\{\{T, P,\{Q, \mathbf{E}\}\},\{f, s, \partial \mathbf{E} / \partial t\}\} \\
\left\{\left\{F \backslash T, S \backslash P,\left\{0, \mathbf{B}^{1}+\mathbf{B}^{2}\right\}\right\},\{\tau, \pi(p), \partial \mathbf{E} / \partial t\}\right\} \\
\left\{\varnothing, \varnothing,\left\{Q, \mathbf{E}+\mathbf{B}^{2}\right\}\right\},\{\varnothing, \varnothing, \partial \mathbf{E} / \partial t\} \\
\left\{\varnothing, \varnothing,\left\{Q, \mathbf{E}+\mathbf{B}^{1}\right\}\right\},\{\varnothing, \varnothing, \partial \mathbf{E} / \partial t\}
\end{array}\right\}
$$

Now we can replace $\partial \mathbf{E} / \partial t$ in the small-scale part of $z_{1}(t, \mathbf{x}, q)$ by a zero field and thus by an empty set, because this field derivative is not compatible with the time observation $f$, describing a static H-event. In the large-scale element of the same zip, no space is available to be marked with $\mathbf{E}$, so the mark set $\{Q, \mathbf{E}\}$ reduces to charge $Q$ attached to $P$. Because the small scale mark element can be replaced by an empty set, the large-scale mark is not observable, so $Q$ has to be written between round brackets. The remaining three zips describe the same interaction, so as a consequence of the incompatibility of mark and time in $z_{1}(t, \mathbf{x}, q)$, we have to replace $\partial \mathbf{E} / \partial t$ in these zips also by an empty set. Hence we met the starting requirements of this section. Because then the small-scale element in zip $z_{2}(t, \mathbf{x}, q)$ is empty, large-scale mark element $\mathbf{B}^{1}+\mathbf{B}^{2}$ is not observable, so it will be placed between round brackets. Zips $z_{3}(t, \mathbf{x}, q)$ and $z_{4}(t, \mathbf{x}, q)$ have empty space observations, so there is nothing to mark; moreover, the time observations are empty, so these zips are empty. Then we obtain the time-space-mark zipper for equally charged coinciding H-units as the set with two elements

$$
Z(t, \mathbf{x}, q)=\left\{\begin{array}{l}
\{\{T, P,(Q)\},\{f, s, \varnothing\}\} \\
\left\{\left\{F \backslash T, S \backslash P,\left(\mathbf{B}^{1}+\mathbf{B}^{2}\right)\right\},\{\tau, \pi(p), \varnothing\}\right\}
\end{array}\right\}
$$

The zipper shows that, by charging the coinciding H-units equally, the resulting two H-events are extended with a charge and a magnetic field, respectively, although both items are not observable. Clearly, by the interaction potential energy is tranformed into actual electromagnetic energy. Because both small scale mark observations (at the right) are zero, this energy can only be stored in spatial objects $P$ and in $S \backslash P$ : The energy of charge $Q$ is stored in point $P$ and the energy of magnetic field $\mathbf{B}^{1}+\mathbf{B}^{2}$ is stored in space $S \backslash P$. In zip $z_{1}(t, \mathbf{x}, q)$, point $P$ can store only mark energy, so this energy is two times the potential energy of one $\mathrm{H}$-unit. In zip $z_{2}(t, \mathbf{x}, q)$, space $S \backslash P$ stores space energy as well as electromagnetic energy; minor particle $\pi(p)$ stores only space energy. Thus a marked $\mathrm{H}$-unit has less potential energy available to generate space than an unmarked $\mathrm{H}$-unit. Assuming that the energetic density of a space is constant, $S$ has to be smaller than in the case of unmarked H-units. We supposed this previously (Spitzer, L. Jr., n.d.), without explaining in detail. Note that the electric field is not represented at all, so this remains a potential feature.

Summarizing the example of coinciding equally charged H-units, zip $z_{1}(t, \mathbf{x}, q)$ describes a charged major particle having an undetermined velocity; zip $z_{2}(t, \mathbf{x}, q)$ describes a major space carrying a magnetic field and an accelerating minor particle upon the major particle. The example shows that the difference between a marked and an unmarked H-unit is not only in the quality 'mark' but also in the quality 'space', resulting from the definition of an H-unit as an elementary amount of potential energy: The potential energy of an uncharged $\mathrm{H}$-unit, in the following indicated by $H_{0 i}$, is entirely available for space features, whilst a marked $\mathrm{H}$-unit, 
indicated by $H_{i}$, has to spend a part of it on electromagnetic features and thus the spatial proportions of $H_{i}$ are reduced with respect to $H_{0 i}$.

In our second paper (Backerra 2012) we suggested that the major particle in this example may be identified as an electron or positron, because of its perfect spherical shape; in the case of opposite charges we identified it as a neutron. However, a neutron is much larger than an electron or positron, so this is only possible if H-units are allowed to have minor spaces with different sizes. At the moment we restrict ourselves still to fixed sizes; more research is required to find out if this requirement may be dropped.

\subsection{The General Mark Zipper}

Above we obtained two more limiting conditions, (49) and (51), besides the two which emerged from the definitions, (36) and (37). The four of them together can be recognized as the laws of Maxwell:

$$
\widetilde{\nabla} \cdot \widetilde{\mathbf{E}}_{i}=\widetilde{Q}_{i} ; \widetilde{\nabla} \cdot \widetilde{\mathbf{B}}^{i}=0 ; \partial \widetilde{\mathbf{E}}_{i} / \partial \tilde{t}=\widetilde{\nabla} \times \widetilde{\mathbf{B}}^{i} ; \partial \widetilde{\mathbf{B}}^{i} / \partial \tilde{t}=-\widetilde{\nabla} \times \widetilde{\mathbf{E}}_{i},(56)
$$

which is an encouraging result. The difference with his formulation, expressed by the tildes, is that Maxwell considered the fields and field derivatives as mathematical and physical descriptions in the same time, whilst we are taking one step more, by transforming the mathematical field items into physical field items. This results in field items which are not infinite, but reach only as far as the major spaces allow. Using these four conditions in specific cases, mark zipper (42), based upon the set of attributes (38), can in general be reduced considerably.

Note that the first condition (36) implies that the mark zipper is largely defined by the two charges $Q_{i}$ and $Q_{j}$. This is analogous to the time zipper, defined by $T_{i}$ and $T_{j}$, and the space zipper, defined by $P_{i}$ and $P_{j}$. For completeness, we show the set of mark attributes $h_{i}(\tilde{q})$ combined with the sets of time and space attributes $h_{i}(\tilde{t})$ and $h_{i}(\tilde{\mathbf{x}})$ (see (38), (16) and (6)), obtaining the set of time-space-mark attributes $h_{i}(\tilde{t}, \tilde{\mathbf{x}}, \tilde{q})$ of $H_{i}$ as

$$
h_{i}(\tilde{t}, \tilde{\mathbf{x}}, \tilde{q})=\left\{\begin{array}{l}
\left\{\widetilde{T}_{i}, \widetilde{P}_{i},\left\{\widetilde{\mathbf{E}}_{i}, \widetilde{Q}_{i}\right\}\right\},\left\{\widetilde{F}^{i} \backslash \widetilde{T}_{i}, \widetilde{S}^{i} \backslash \widetilde{P}_{i},\left\{\widetilde{\mathbf{B}}^{i}, \widetilde{Q}_{i} \times i\right\}\right\}, \\
\left\{\tilde{\tau}_{i}, \tilde{p}_{i}, \widetilde{\nabla}_{i}\right\},\left\{\widetilde{f}^{i}, \tilde{s}^{i}, \partial / \partial \tilde{t}^{i}\right\}
\end{array}\right\} .
$$

When considering a specific case, it is often practical to derive the zippers for each of the qualities time, space and mark separately, resulting in more manageable sets of observations. By combining them, usually some more elements will reduce to empty sets, because of being incompatible with elements of another quality. Even if electromagnetic items are compatible with time observations, they still have to be restricted to the available space, because the mathematical fields are defined as infinite, whilst the zipper contains only finite spaces. The storage of electromagnetic energy requires a space carrying vector fields, or a point of space carrying a charge. Up to now we only found zippers that finally contained only two non-empty elements.

\section{Photons}

When deriving elementary particles (Backerra, 2012), we found that, to describe a moving elementary particle, the involvement of at least one extra unmarked $\mathrm{H}$-unit $H_{0 i}$ is required. When describing gravity (Backerra 2014) we found out that two unmarked H-units were necessary as a background for charged H-units. In this paper we want to describe a photon, being a massless electromagnetic item which can travel over a huge distance. To achieve this, we need at least one unmarked H-unit $H_{01}$ to supply a large space, and one marked H-unit $H_{2}$ to supply electromagnetic features. We have to be aware of the fact that $\mathrm{H}_{2}$ has smaller spatial dimensions than $H_{01}$, as is explained above. For convenience we suppose the difference such that $\widetilde{S}^{01}>>\widetilde{S}^{2}$. Next we have to choose a space case which might give us a description of a photon.

In previously papers we discovered that mass is generated by $\mathrm{H}$-units with intersecting minor spaces and pellicles, so now we will choose their mutual distance such that minor spaces and pellicles have empty intersections. On the other hand, the H-units must be close enough to be able to interact, so their major spaces must have a non-empty intersection. Because $\underset{\sim}{\text { we }}$ would like to have a zipper describing a localized major point, we choose them such that $\widetilde{P}_{01} \in \widetilde{S}^{2}$ and $\widetilde{P}_{2} \in \widetilde{S}^{01}$. If the radius of $\tilde{S}^{2}$ is called $r_{2}$ and the radius of $\widetilde{S}^{2}$ is $R_{2}$, this condition can be written as $r_{2}<\left|\widetilde{P}_{2}-\widetilde{P}_{01}\right|<R_{2}$. Mind that we cannot obtain a major point of space if $\left|\widetilde{P}_{2}-\widetilde{P}_{01}\right|=R_{2}$, because the border of $\tilde{S}^{2}$ is by definition excluded. Because we want to deal with time in an analogous way as with space, we choose the difference in major points of time similarly. Thus $\left|\widetilde{T}_{2}-\widetilde{T}_{01}\right|>\tilde{t}+d \tilde{t}$, in which $\tilde{t}$ is the width of $\widetilde{f}$ and $\tilde{\tau}^{01}$ is the width of $\tilde{\tau}$, with $\tilde{t}=\tilde{t}_{01}=\tilde{t}_{2}$, $d \tilde{t}=d \tilde{t}_{01}=d \tilde{t}_{2}$ and $\widetilde{f}=\widetilde{f}^{01}=\widetilde{f}^{2}$. This implies that we have two time cases: $\widetilde{T}_{01}-\widetilde{T}_{2}>\tilde{t}+d \tilde{t}$ and $\widetilde{T}_{2}-\widetilde{T}_{01}>\tilde{t}+d \tilde{t}$. As opposed to space attributes, the size of time attributes is not affected by marking because 
they do not store energy, so they are equal for marked and unmarked $\mathrm{H}$-units. We will deduce the time-space-mark zipper for this case.

When an unmarked $\mathrm{H}$-unit $H_{01}$ interacts with a marked one $H_{2}$, the mark zipper is obtained by dropping the not-existing fields and charges of $H_{01}$ in (42), so

$$
Z(q)=\left\{\begin{array}{l}
{\left[\left\{\widetilde{Q}_{2}, \widetilde{\mathbf{E}}_{2}\right\}, \partial \widetilde{\mathbf{E}}_{2} / \partial \tilde{t}\right]} \\
{\left[\left\{\widetilde{Q}_{2} \times i, \widetilde{\mathbf{B}}^{2}\right\}, \widetilde{\nabla} \times \widetilde{\mathbf{B}}^{2}\right]} \\
{\left[\left\{\widetilde{Q}_{2} \times i, \widetilde{\mathbf{B}}^{2}\right\}, \widetilde{\nabla} \times \widetilde{\mathbf{B}}^{2} \propto \partial \widetilde{\mathbf{B}}^{2} / \partial \tilde{t}\right]} \\
{\left[\left\{\widetilde{Q}_{2}, \widetilde{\mathbf{E}}_{2}\right\}, \partial \widetilde{\mathbf{E}}_{2} / \partial \tilde{t} \propto \widetilde{\nabla} \times \widetilde{\mathbf{E}}_{2}\right]}
\end{array}\right\}
$$

For $\widetilde{T}_{01}-\widetilde{T}_{2}>\tilde{t}+d \tilde{t}$, the time zipper, using (34), is

$$
Z(t)=\left\{\{\varnothing, \varnothing\},\left\{F^{01} \backslash T_{01} \cap F^{2}, \varnothing\right\},\left\{T_{01}, \varnothing\right\},\{\varnothing, \varnothing\}\right\}
$$

Note that the part of the time axis from $T_{01}$ to $T_{2}$, which could be considered as the past, is not represented.

For $\widetilde{T}_{2}-\widetilde{T}_{01}>\tilde{t}+d \tilde{t}$ the time zipper is

$$
Z(t)=\left\{\{\varnothing, \varnothing\},\left\{F^{01} \cap F^{2} \backslash T_{2}, \varnothing\right\},\{\varnothing, \varnothing\},\left\{T_{2}, \varnothing\right\}\right\},
$$

so in this case the time interval from $T_{2}$ to $T_{01}$ is not represented. The difference between time zippers (59) and (60) is, that in the first time case $z_{3}(t)$ describes actual energy and $z_{4}(t)$ potential energy; in the second time case this is reversed. Because these two time cases describe the same interaction between H-units, to obtain a full description of the generated $\mathrm{H}$-events both have to be considered.

Inserting the attributes of space in (35), we obtain space zipper

$$
Z(\mathbf{x})=\left\{\{\varnothing, \varnothing\},\left\{S^{01} \cap S^{2}, \varnothing\right\},\left\{P_{01}, \varnothing\right\},\left\{P_{2}, \varnothing\right\}\right\} .
$$

Note that we obtain one three-dimensional description for the space-zipper, whilst there are two one-dimensional time zippers.

\subsection{The First Time Case}

First we consider this case for $\widetilde{T}_{01}-\widetilde{T}_{2}>\tilde{t}+d \tilde{t}$. Combining time zipper (59) with space zipper (61) and mark zipper (58), we obtain the time-space-mark zipper

$$
Z(t, \mathbf{x}, q)=\left\{\begin{array}{l}
{\left[\left\{\varnothing, \varnothing,\left\{\widetilde{Q}_{2}, \widetilde{\mathbf{E}}_{2}\right\}\right\},\left\{\varnothing, \varnothing, \partial \widetilde{\mathbf{E}}_{2} / \partial \tilde{t}\right\}\right]} \\
{\left[\left\{\widetilde{F}^{01} \backslash \widetilde{T}_{01} \cap \widetilde{F}^{2}, \widetilde{S}^{2},\left\{\widetilde{Q}_{2} \times i, \widetilde{\mathbf{B}}^{2}\right\}\right\},\left\{\varnothing, \varnothing, \widetilde{\nabla} \times \widetilde{\mathbf{B}}^{2}\right\}\right]} \\
{\left[\left\{\widetilde{T}_{01}, \widetilde{P}_{01},\left\{\widetilde{Q}_{2} \times i \propto \widetilde{\mathbf{B}}^{2}\right\}\right\},\left\{\varnothing, \varnothing, \widetilde{\nabla} \times \widetilde{\mathbf{B}}^{2} \propto \partial \widetilde{\mathbf{B}}^{2} / \partial \tilde{t}\right\}\right]} \\
{\left[\left\{\varnothing, \widetilde{P}_{2},\left\{\widetilde{Q}_{2}, \widetilde{\mathbf{E}}_{2}\right\}\right\},\left\{\varnothing, \varnothing, \partial \widetilde{\mathbf{E}}_{2} / \partial \tilde{t} \propto \widetilde{\nabla} \times \widetilde{\mathbf{E}}_{2}\right\}\right]}
\end{array}\right\}
$$

After transforming them, all mark attributes have to be compatible with the corresponding space attributes. In zip $z_{1}(t, \mathbf{x}, q)$ these are empty, so this zip can be replaced by an empty set. Zip $z_{4}(t, \mathbf{x}, q)$ contains no time attributes at all, so its energy stays potential and it can also be replaced by an empty set. Thus $z_{1}(t, \mathbf{x}, q)$ and $z_{4}(t, \mathbf{x}, q)$ will be dropped. Zips $z_{2}(t, \mathbf{x}, q)$ and $z_{3}(t, \mathbf{x}, q)$ contain non-zero time labels, so they describe actual phenomena. Both small-scale time and space observations (at the right) are empty, so the large-scale time and space observations (at the left) are not observable and have to be placed between round brackets. In zip 
$z_{2}(t, \mathbf{x}, q)$, left time label $\left(F^{01} \backslash T_{01} \cap F^{2}\right)$ indicates that the zip describes actual energy; because the small scale time label is empty, the described physical item is not observable.

The imaginary number in the large-scale mark element of $z_{3}(t, \mathbf{x}, q)$ transforms to zero. In zip $z_{3}(t, \mathbf{x}, q)$, large-scale time label $T_{01}$ indicates that actual energy is described, because the small-scale time label is empty, $T_{01}$ is not observable. Large-scale space element $P_{01}$ indicates that $P_{01}$ is actual existing; it is not observable because the small scale space element is empty. By transforming the remaining elements straight forward, equation (62) reduces to the first time-space-mark zipper

$$
Z(t, \mathbf{x}, q)=\left\{z_{2}, z_{3}\right\}=\left\{\begin{array}{l}
\left\{\left\{\left(F^{01} \backslash T_{01} \cap F^{2}\right),\left(S^{01} \cap S^{2}\right),\left\{0, \mathbf{B}^{2}\right\}\right\},\left\{\varnothing, \varnothing, \nabla \times \mathbf{B}^{2}\right\}\right\} \\
\left\{\left\{\left(T_{01}\right),\left(P_{01}\right),\left\{0, \mathbf{B}^{2}\right\}\right\},\left\{\varnothing, \varnothing, \nabla \times \mathbf{B}^{2}+\partial \mathbf{B}^{2} / \partial t\right\}\right\}
\end{array}\right\}
$$

The energy of zip $z_{2}(t, \mathbf{x}, q)$ is divided over both space and electromagnetism. The observation $\nabla \times \mathbf{B}^{2}$ is not accompagnied by time and space elements, but because the large-scale observation does contain elements of time and space, it is compatible and thus $\nabla \times \mathbf{B}^{2}$ is actual existing. The absence of small-scale time and space observations indicates that $\nabla \times \mathbf{B}^{2}$ is independent of time and space, as far as the large-scale observations of time and space allow. The fields were mathematically defined as infinite mark fields, so the zip acts as a restriction of the magnetic field derivation to interval of time $F^{01} \backslash T_{01} \cap F^{2}$ and space $S^{01} \cap S^{2}$; consequently, also the electric and magnetic fields and all derivatives are restricted to the overlapping regions of time and space.

Space independency of $\nabla \times \mathbf{B}^{2}$ implies, that the field exists in the complete spatial region of interaction; time independency implies that $\partial\left(\nabla \times \mathbf{B}^{2}\right) / \partial t=\mathbf{O}$. Because of field condition (49), $\nabla \times \mathbf{B}^{2}$ is equal to $\partial \mathbf{E}_{2} / \partial t$, so $\partial^{2} \mathbf{E}_{2} / \partial t^{2}=\mathbf{O}$ and thus the electric field is restricted by the condition

$$
\partial \mathbf{E}_{2} / \partial t=\mathbf{C}
$$

in which $\mathbf{C}$ is a constant field. This implies that $P_{2}$ moves with a constant velocity. This is possible in two ways: by circling around $P_{01}$ or by moving to or from $P_{01}$. First we consider the most stable description, which seems to be the circling; obviously this implies that $P_{2}$ also is circling with a constant velocity around $P_{01}$. Zip $z_{2}(t, \mathbf{x}, q)$ describes observable magnetic fields $\mathbf{B}^{2}$ and $\nabla \times \mathbf{B}^{2}$ in the unobservable common future and the unobservable major space $S^{01} \cap S^{2}$. This shows that the zip acts as a restriction of these fields, which are mathematically infinite. Thus the actual energy of $z_{2}(t, \mathbf{x}, q)$ is divided over unobservable space energy and observable electromagnetic energy. Zip $z_{3}(t, \mathbf{x}, q)$ describes only electromagnetic energy, which can be stored only in $P_{01}$. To this point are attached two electromagnetic items: the complete electromagnetic field $\nabla \times \mathbf{B}^{2}+\partial \mathbf{B}^{2} / \partial t$, being independent of time and space (because of the absence of small scale time and space observations) and existing in the overlapping regions of time and space, and a vector of $\mathbf{B}^{2}$, indicated by $\mathbf{b}^{2}$, being independent of time and space (because of (64)). So the large scale mark element can be reduced to $\left\{0, \mathbf{b}^{2}\right\}$. At first sight it might be surprising that an electromagnetic field is carried by one physical point; on the other hand, in the past an electromagnetic field was supposed to reach to infinity, even without a physical point of attachment.

All together, zip $z_{3}(t, \mathbf{x}, q)$ describes a neutral, massless point particle at $P_{01}$ and $T_{01}$, both items being not observable, storing electromagnetic energy by carrying magnetic vector $\mathbf{b}^{2}$ and the entire electromagnetic field $\nabla \times \mathbf{B}^{2}+\partial \mathbf{B}^{2} / \partial t$, existing in the overlapping regions of time and space. The particle circles with a constant velocity around $P_{2}$; thus it acts as a clock and time is running. Because the major space may have an astronomic radius, the track of the photon might resemble a straight line. Because the distance to $P_{2}$ plays no role in the expression, the velocity of the massless particle is independent of the mutual distance, so the distance has no influence upon the energy of the point particle. The generated particle can be identified as a photon, the elementary particle without mass and charge, having an unobservable major location and carrying observable electromagnetic energy through space with a constant velocity. Its path is circular, so, as described in the theory of general relativity, the photon follows a curved path. In section 8.4 we will consider its stability.

Second we consider the possibility that $P_{2}$ moves to or from $P_{01}$. If they move to each other, this case is finished as soon as the minor spaces of $\mathrm{H}_{01}$ and $\mathrm{H}_{2}$ touch each other. From that moment the energy of H-units can be spent on mass, which is identified as the absorption of the photon. In case they move from each other, the photon will annihilate as soon as the major spaces do not overlap anymore; then the H-units stop to interact and the electromagnetic energy will be transformed back to potential energy. These two events are not cyclic and thus time is not running. 
Summarizing, the second time case gives the description of a particle which can be identified as a photon, circling or moving straight. Because we know experimentally that photons may travel during years, H-units may have major spaces with a radius of astronomic size.

\subsection{The Second Time Case}

Second we consider this case for $\widetilde{T}_{2}-\widetilde{T}_{01}>\tilde{t}+d \tilde{t}$. Combining time zipper (60) with space zipper (61) and mark zipper (58) and reducing it in similar way as above, we obtain the second time-space-mark zipper

$$
Z(t, \mathbf{x}, q)=\left\{z_{2}, z_{4}\right\}=\left\{\begin{array}{l}
\left\{\left\{\left(F^{01} \cap F^{2} \backslash T_{2}\right),\left(S^{01} \cap S^{2}\right), \mathbf{B}^{2}\right\},\left\{\varnothing, \varnothing, \nabla \times \mathbf{B}^{2}\right\}\right\} \\
\left\{\left\{\left(T_{2}\right),\left(P_{2}\right),\left\{Q_{2}, \mathbf{E}_{2}\right\}\right\},\left\{\varnothing, \varnothing, \partial \mathbf{E}_{2} / \partial t+\nabla \times \mathbf{E}_{2}\right\}\right\}
\end{array}\right\}
$$

Zip $z_{1}(t, \mathbf{x}, q)$ again is empty and zip $z_{3}(t, \mathbf{x}, q)$ contains no time attributes, so they can be dropped. Zip $z_{2}(t, \mathbf{x}, q)$ describes the same as in the previous case, with interchanged major points of time, so again this zip acts as a restriction to the described electromagnetic fields and their derivations. Similar as we derived above, $P_{01}$ and $P_{2}$ circle around each other with a constant velocity. The new item is $z_{4}(t, \mathbf{x}, q)$. The large-scale time label $T_{2}$ indicates that actual energy is described; the round brackets indicate that $T_{2}$ is not observable, because the small-scale time label is empty. The left space observation describes $P_{2}$ as an actual point of space; the round brackets indicate that $P_{2}$ is not observable, because the right space element is empty. In $P_{2}$ no space energy can be stored and there is no minor space object described, so $z_{4}(t, \mathbf{x}, q)$ can represent only electromagnetic energy, stored in $P_{2}$, described by two items. The large scale electromagnetic observation $\left\{Q_{2}, \mathbf{E}_{2}\right\}$ reduces to $\left\{Q_{2}, \mathbf{e}\right\}$. According to the small scale mark element, the electromagnetic field $\partial \mathbf{E}_{2} / \partial t+\nabla \times \mathbf{E}_{2}$ is time and space independent, only restricted to the overlapping time and space observations, according to $z_{2}(t, \mathbf{x}, q)$. All together zip $z_{4}(t, \mathbf{x}, q)$ describes a charged, massless point particle at $P_{2}$ and $T_{2}$, both items being not observable, storing electromagnetic energy by carrying charge $Q_{2}$, electric vector $\mathbf{e}$ and the entire electromagnetic field $\partial \mathbf{E}_{2} / \partial t+\nabla \times \mathbf{E}_{2}$, existing in the overlapping regions of time and space. Again this is possible in two ways, circling around $P_{01}$ or by moving to or from $P_{01}$.

If the particle circles with a constant velocity around $P_{01}$, it acts as a clock and time is running; the major space may have an astronomic radius, so its track might resemble a straight line. Because the distance to $P_{01}$ plays no role in the expression, the velocity of the particle is independent of the distance to $P_{2}$, so this has no influence upon the energy of the point particle. The point particle is generated by the same interaction as above, where a photon is generated in the opposite time case. As the photon carries an imaginary charge (see zipper (62) and (63)), the charged item may be considered as the anti-particle of the photon. Thus we identify the zip as an anti-photon, a particle without mass, carrying an observable charge at a unobservable major location, and an observable electromagnetic field energy. We will indicate the anti-photon by $\gamma^{q}$, with $q$ indicating its charge; consequently, the photon will be indicated by $\gamma^{0}$, with 0 indicating zero charge. Apparently they constitute a new type of particle-pair, the photon-pair. Well-known particle-pairs have real, opposite charges and they move in opposite directions. In this case they have opposite charges in complex sense and the circling around each other can be considered as another type of opposite movement.

If $P_{01}$ and $P_{2}$ move to each other with a constant velocity, than the anti-photon annihilates as soon as the minor spaces of $\mathrm{H}_{01}$ and $\mathrm{H}_{2}$ touch each other, or as soon as the major spaces do not overlap each other anymore.

It is common experimental knowledge that the generation as well as annihilation of a photon is accompanied by an accelerated charge. Accelerated charge is even the only constant feature of the origin and termination of an EM wave and usually it is considered as the origin of the photon. It is striking that we find a charged particle as the anti-particle of the photon. However, at first sight the charge seems not to be accelerating. In classical mechanics the circling of any object requires a force perpendicular to its track, to consider the object as accelerated and at an atomic level, this notion is generalized by supposing that any circling item can be considered as accelerated. In twin physics, on the contrary, a physical item may circle around with a constant velocity without introducing acceleration, because the spaces are defined as spherical objects. Thus the circling charge as described above, accompagnying the photon as its anti-particle, might be the same item as the accelerated charge, in another context considered as the origin of photons.

Note that, if we had combined space and time to a four-dimensional quality of H-units, we would have obtained only one zipper and presumably this would have been not enough to describe a photon-pair, because a third zip is necessary to restricts the infinite mark fields to finite electromagnetic fields and up to now, zippers contain maximum two H-events. 


\section{The Energy of a Photon}

In this section we consider the energy of the photon. Subsequently, the exchange of energy of a photon-pair, the transport of energy through space, quantization of energy and annihilation will be considered.

\subsection{Exchange of Energy}

Although the two H-events $\gamma^{0}$ and $\gamma^{q}$ exist simultaneously, they cannot be observed simultaneously, as they are described by distinct zips. Nevertheless, a remarkable feature about their connection is hidden in their zips.

If, for convenience, we repeat zip $z_{3}(t, \mathbf{x}, q)$ of Equation (63),

$$
z_{3}(t, \mathbf{x}, q)=\left\{\left\{\left(T_{01}\right),\left(P_{01}\right),\left\{0, \mathbf{B}^{2}\right\}\right\},\left\{\varnothing, \varnothing, \nabla \times \mathbf{B}^{2}+\partial \mathbf{B}^{2} / \partial t\right\}\right\}
$$

and compare this with $z_{4}(t, \mathbf{x}, q)$ of Equation (65), which is

$$
z_{4}(t, \mathbf{x}, q)=\left\{\left\{\left(T_{2}\right),\left(P_{2}\right),\left\{Q_{2}, \mathbf{E}_{2}\right\}\right\},\left\{\varnothing, \varnothing, \partial \mathbf{E}_{2} / \partial t+\nabla \times \mathbf{E}_{2}\right\}\right\},
$$

it is striking that both zips contain an actual small-scale electromagnetic observation not only in points $P_{01}$ and $P_{2}$, respectively, but in the total region of interaction, because these items are space independent. In $z_{3}(t, \mathbf{x}, q)$ the actual field $\nabla \times \mathbf{B}^{2}+\partial \mathbf{B}^{2} / \partial t$ is observable because it is at point of time $T_{01}$ attached to point of space $P_{01}$, and because this field is time and space independent, it is also actual (but not observable) in the remaining part of the overlapping space of the H-units, including $P_{2}$. Similarly, the actual field $\partial \mathbf{E}_{2} / \partial t+\nabla \times \mathbf{E}_{2}$ in $z_{4}(t, \mathbf{x}, q)$ is observable because it is at point of time $T_{2}$ attached to point of space $P_{2}$, and because this field is time and space independent, it is also actual (but not observable) in $P_{01}$. This reveals an immediate exchange of energy between the two H-events, $\gamma^{0}$ being dressed up with an electric field and $\gamma^{q}$ with a magnetic field, which can be identified as the entanglement of electric and magnetic fields to an electromagnetic field and their continuous exchange of energy.

\subsection{Transport of Energy}

Because both time cases label the same interaction, zips $z_{3}(t, \mathbf{x}, q)$ and $z_{4}(t, \mathbf{x}, q)$ above describe the same amount of energy. For both this is only electromagnetic energy, transported through space by $P_{01}$ and $P_{2}$, respectively, as they circle around each other or move to or from each other with a constant velocity $c$. The requirement for a constant velocity for an arbitrary field function $\mathbf{F}(t, \mathbf{x})$ is

$$
\partial \mathbf{F}(t, \mathbf{x}) / \partial t=c \times \partial \mathbf{F}(t, \mathbf{x}) / \partial \mathbf{x} .
$$

Zip $z_{3}(t, \mathbf{x}, q)$ in Equation (66) contains a large-scale vector $\mathbf{b}^{2}$, of which we know that its time and space

derivations are zero (as is explained after Equation (64). So we insert only the small scale electromagnetic observation of (66) in equation (68), and obtain

$$
\partial\left(\nabla \times \mathbf{B}^{2}+\partial \mathbf{B}^{2} / \partial t\right) / \partial t=c \times \partial\left(\nabla \times \mathbf{B}^{2}+\partial \mathbf{B}^{2} / \partial t\right) / \partial \mathbf{x}
$$

which can be reduced by using the field conditions to

$$
\partial\left(\partial \mathbf{E}_{2} / \partial t+\partial \mathbf{B}^{2} / \partial t\right) / \partial t=c \times \partial\left(\partial \mathbf{E}_{2} / \partial t+\partial \mathbf{B}^{2} / \partial t\right) / \partial \mathbf{x}
$$

so to

$$
\partial^{2} \mathbf{E}_{2} / \partial t^{2}+\partial^{2} \mathbf{B}^{2} / \partial t^{2}=c \times\left(\partial^{2} \mathbf{E}_{2} / \partial t \partial \mathbf{x}+\partial^{2} \mathbf{B}^{2} / \partial t \partial \mathbf{x}\right)
$$

Considering $z_{4}(t, \mathbf{x}, q)$ in Equation (67), charge $Q_{2}$ adds an amount of energy to the field energy $\partial \mathbf{E}_{2} / \partial t+\nabla \times \mathbf{E}_{2}$. Because the energy of charge is a constant, it will disappear in the derivations, so we can ignore this large-scale observation. Inserting the small-scale electromagnetic observation of (67) in equation (68), we obtain

$$
\partial\left(\partial \mathbf{E}_{2} / \partial t+\nabla \times \mathbf{E}_{2}\right) / \partial t=c \times \partial\left(\partial \mathbf{E}_{2} / \partial t+\nabla \times \mathbf{E}_{2}\right) / \partial \mathbf{x} .
$$


By using the fieldconditions, we obtain

$$
\partial^{2} \mathbf{E}_{2} / \partial t^{2}-\partial^{2} \mathbf{B}^{2} / \partial t^{2}=c \times\left(\partial^{2} \mathbf{E}_{2} / \partial t \partial \mathbf{x}-\partial^{2} \mathbf{B}^{2} / \partial t \partial \mathbf{x}\right) .
$$

Equations (71) and (73) stay valid if we add their left and right parts, resulting in

$$
\partial^{2} \mathbf{E}_{2} / \partial t^{2}=c \times \partial^{2} \mathbf{E}_{2} / \partial t \partial \mathbf{x}
$$

which can be written as

$$
\partial\left(\partial \mathbf{E}_{2} / \partial t\right) / \partial t=c \times \partial\left(\partial \mathbf{E}_{2} / \partial t\right) / \partial \mathbf{x} .
$$

Similar, by subtracting both left and right parts of (71) and (73) we obtain

$$
\partial\left(\partial \mathbf{B}^{2} / \partial t\right) / \partial t=c \times \partial\left(\partial \mathbf{B}^{2} / \partial t\right) / \partial \mathbf{x} .
$$

According to the two equations above, the actual energetic propagation through space occurs with a constant velocity $c$, which is identified as the velocity of light.

\subsection{Quantization of Energy}

To obtain an expression for the energy of a photon, we have to realize that the interaction is independent of the distance between the photon and the anti-photon, so the generation of energy is also independent of this distance. Thus in case the photon is circling around the anti-photon, its energy can only be dependent of the frequency of circling, indicated by $v$. If we choose the largest possible time of one cycle as a natural unity of time, than the energy of the photon is a constant which can be identified as Planck's constant $h$. This implies that, if $v>1$, than $v$ can only be an integer, and, because the velocity of the photon is constant, the distance must be smaller. The interaction is supposed to be more intense when the H-units are closer, so in that case more energy will be transformed and the energy of the photon will be higher. The lowest border is reached for frequency $v_{n}=1$, when $P_{01}$ and $P_{2}$ are as distant as possible (without loosing contact with each other's major spaces). The upper border is reached if the minor spaces are as close as possible (without overlapping each other).

The remaining question is the dependence of the photon energy on the frequency in the region between the lowest and highest possible energy. Because the time of one cycle at the lowest frequency is the unity of time and thus the frequency is an integer, and the velocity is constant, the energy of the photon and the anti-photon is quantized. The lowest level is $h$, so it is obvious to suppose a linear relationship as

$$
E=h \times v .
$$

This is the famous equation as was introduce intuitively by Max Planck in 1900 (The Editors of Encyclopaedia Brittannica, 1900), to explain black body radiation, and which agrees with many experimental results. It expresses that energy transfer by an electromagnetic field is quantized. In the rest of his life, Planck was not able to deduce this theoretically; apparently twin physics can give a solution. In many cases it is practical to combine $h$ with $2 \pi$ to the common expression $\hbar=h / 2 \pi$, the Dirac constant or the reduced Planck constant. Here, when a photon in $P_{01}$ travels one cycle around $P_{2}$, it covers a distance of $2 \pi \times R \quad$ ( $R$ being their distance). With velocity $c$, the elapsed time is $(2 \pi \times R) / c$. Thus the frequency of the photon is $v=c /(2 \pi \times R)$, which has to be an integer. The essential point is, that in this expression $R$ cannot act as a variable; it is only a specific, constant distance out of a series of distances, each belonging to one specific frequency. If for frequency $v_{n}$ this distance is $R_{n}$ and we insert this in (77), the energy of photon $\gamma_{n}$ can be written as

$$
E_{n}=h \times c /\left(2 \pi \times R_{n}\right),
$$

which contains the expression $\hbar=h / 2 \pi$.

For radio waves with the extreme low frequency of $1 \mathrm{~Hz}$ the distance $\mathrm{R}$ is about $48,000 \mathrm{~km}$, whereas for gamma rays with the extreme high frequency of $3.10^{20} \mathrm{~Hz}, \mathrm{R}$ is $1.6 \times 10^{-13} \mathrm{~m}$ (Elert, G., (1998-2015)). This shows that an $\mathrm{H}$-unit indeed connects the extremes in dimensions by having in its set of space attributes a major as well as a minor space.

Looking back to the derivation of the zipper for interacting H-units, one being neutral and the other being charged, we can describe the results in a more poetical way by saying that charge can give birth to a photon-pair 
by interacting with a neutral H-unit. These massless particles, not being able to rest, can be considered as the dynamic manifestations of energy in time. By cyclic movement, a natural unity of time is generated and thus $\boldsymbol{a}$ time axis develops. The amount of cycles per second determines their energy, in that way betraying the existence of space. This means that the circling photon-pair is the key between space and time.

\subsection{The Term of a Photon}

We considered two ways in which photons can move: circling or in a straight line. If it moves to or from the anti-photon, obviously it will annihilate as soon as the pellicles of the $\mathrm{H}$-units touch each other or the major spaces do not overlap anymore, because then the case will be finished. The circling possibility seems the most stable one, but when we take a closer look, possibly this is not an infinite stability because the circling photon acts as a clock and the time is running. In daily life we know that because of the running of time, all processes have a finite duration, even the stars, so we want to find out if the interaction between the two H-units in this case also has a finite duration of life. As the major points of time $T_{01}$ and $T_{2}$ move over the time axis as the time runs, they take the three remaining time attributes with them, but in each of the two time cases an obstacle prevents this to go on infinitely. This obstacle is $T_{e 1}$ being the end of the span $\widetilde{F}^{1}$, and $T_{e 2}$ being the end of the span $\widetilde{F}^{2}$, respectively. As defined in Equation (17), $T_{e i}$ is excluded from $\widetilde{F}^{i}$, so it is not involved in the interaction; it is merely a fixed point upon the time axis and there is no reason why it should move together with $T_{01}$ and $T_{2}$. This implies that, as the photon-pair continues to circle around each other, $T_{e 1}$ will come closer to $T_{01}$ and $T_{e 2}$ will come closer to $T_{2}$. Thus the future $F^{01} \backslash T_{01} \cap F^{2}$ of the photon will shrink, as well as the future $F^{01} \cap F^{2} \backslash T_{2}$ of the anti-photon. Because time and space are treated in exactly the same way, we assume that $S^{01} \cap S^{2}$ will shrink as well. Because zip $z_{2}(t, \mathbf{x}, q)$ acts as a restriction to the extent of the electromagnetic fields, the fields are extended over a shrinking space and thus the series of possible distances between the photon and the anti-photon $R_{n}$ will have a decreasing maximum in steps. So if the photon-pair exists at the largest possible distance, after some time it cannot circle around anymore; the only possibility to continue, is moving towards each other. As soon as they reach a lower level of distance, they may again circle around each other and if they do so, the frequency is increased because of their constant velocity, so their energy is increased. Thus in each step to a smaller radius, the energy of the photon-pair increases, which is a remarkable consequence because we know photons experimentally only as having a constant frequency. Finally the major spaces of the $\mathrm{H}$-units will be of the order of magnitude of the minor spaces. Then the pellicles will touch each other, so this case is finished, the photon-pair annihilates and time stops to run. Each of the two H-units still contains their constant amount of energy, but it cannot be transformed into a large space any more. In principle this energy can be used to generate mass or it transforms back to potential energy.

These two consequences of the shrinking of the future, the annihilation of the photon-pair and the time stopping to run, could be responsible for the two astronomic phenomena dying stars and black holes. If a photon-pair continues to circle as long as possible, being highly energetic, it might be involved in the explosion generating many chemical compounds which is characteristic for a dying star (Spitzer, L. Jr., n.d.). If the photon-pair moves towards each other, it might be involved in the generation of massive particles and these cannot be more heavy than that of the major particle, as described for unmarked coinciding H-units (see Equation (24)), because the maximum of its energy is the sum of the potential energy of two H-units. But there is an important difference, because they are surrounded by major spaces which are shrinked down to the proportions of minor spaces, so they can be considered as bald. Consequently many of these particles may exist extremely close to each other without any possibility to interact with each other and the time stands still. In this way an object may exist having an extreme large density of mass, identified as a black hole (Smith, 2008).

\subsection{A Renewed Concept of Energy}

The law of conservation of energy is anchored in the basics of twin physics. This is realized by considering an $\mathrm{H}$-unit as a elementary unity of potential energy and introducing the zipper as the set of all possible transformations to actual energy, by interaction with another $\mathrm{H}$-unit. According to twin physics, the law of conservation of energy can be extended to the law of conservation and transformation of energy.

We considered two extreme cases of transforming energy from potential to actual, for coinciding H-units and for $\mathrm{H}$-units at a maximal distance. In the coinciding case, the result is a static mass in a large space. The existence of time is betrayed only during an infinitesimal period of time by an accelerating minor particle upon its surface. In the distant case, the result is a photon with its anti-particle, transporting energy with the highest possible velocity, the velocity of light, without showing its position. Mind that we know its position only at the moment the photon is absorbed. The existence of space is betrayed only by the cyclic character of the movement with respect to the anti-photon, because its energy is determined by the amount of cycles per second. So in the first example the energy of the interacting H-units is expressed as mass and in the second as a clock. 
In the light of these two examples, we distinguish two types of potential energy: static and dynamic potential energy. Static potential energy is conceived as the potential power to generate presence. If static potential energy is transformed, space energy is generated, stored as a mass (having a high energetic density) or as a large surrounding space (having a low energetic density). Dynamic potential energy is conceived as the potential power to generate difference. Potential difference in $\mathrm{H}$-units can be provided by marking their spaces and points. If dynamic potential energy is transformed, electromagnetic energy is generated, stored as an electromagnetic field in a space or as charge in a point. We expect this classification of potential energy to be helpful for the comprehension of energy.

\section{Conclusion}

We considered two cases, each concerning the interaction of two Heisenberg units, representing two extremes in spatial sense. In the first case, two H-units coincide generating mass, which is the most easy physical item to imagine. In the second case, two H-units had a distance too large to generate mass; they generate a photon, which is the most difficult item to imagine because it is unobservable as long as it is not absorbed. In deriving these cases, it turned out to be useful to follow Einstein's suggestion to deal with time and space in a similar way. However, their combination into a four-dimensional continuum would not have led to a description of the photon.

In the second case, not only a photon was produced but also its anti-particle, called the anti-photon. This new particle is also unobservable, as long as it is not absorbed, and it carries a charge, whilst a photon is neutral. Both particles travel around each other at a constant velocity, which can be identified as the velocity of light. The largest time for one cycle to be completed is chosen as the natural unit of time and this is used to generate a time axis. As a consequence, the frequency of the photon is an integer and its energy is quantized. The photon energy with the lowest possible frequency is identified as Planck's constant. By considering the photon-pair more close, a possible explanation of the development of dying stars and black holes can be deduced.

Our complementary way of thinking, supplied with the notion of major and minor influences, leads to a reconciliation of quantum mechanical and relativity theory. This implies that twin physics is not only suitable for describing gravity, as we explained in our previous paper (Backerra, 2014), but also provides in a bridge between subatomic and astronomic items in general.

Thus we conclude that the H-unit, defined as an elementary unity of potential energy and expressed in complementary terms, can be considered to be fundamental in nature. Its mathematical attributes allow the combination of subatomic and astronomic distances in a manageable way, without the losing any information due to the huge difference in order of magnitude.

In our theory, the concept of time is expanded from purely deterministic to complementary, thus incorporating the uncertainty of time. As a consequence time no longer runs on its own as it was supposed to do in classical physics; time exists only as long as the H-events interact with each other. Time is running only if a cyclic phenomenon is generated and if not, it stands still. Without interaction, time even does not exist. Time in twin physics is not an energetic object, but a label of interaction, indicating how potential energy is transformed into actual energy, in a static or a dynamic form, determinate or indeterminate.

Our energetic concept of space reflects uncertainty such that it implies a new and inescapable concept of probability. It has the exciting consequence that any influence upon a space will exist instantaneously everywhere, which may have severe philosophical consequences. Although it resembles the historical notion of 'ether', there are two important differences. The first one is that space, according to our definition, is not infinite but limited. The second is that space is not defined as a collection of determinate spatial points, but as a collection of indeterminate spatial points of which each point is conceived as stretched out over the entire available space.

In this paper, together with the previous papers (Backerra, 2010, 2012,2014), we have exhaustively investigated the interaction between two H-units. In some cases a third, a fourth or many H-unit are involved, but only with respect to their major spaces. It will be very interesting to seek for a description of the interaction between more than two H-units in detail. The most intriguing question is whether twin physics can be expanded to systems of many H-units, and how this can be done.

\section{Acknowledgments}

I am very grateful to Willem van Erk for our essential discussions and his thorough correction of the manuscript, Wim Graef for our fundamental discussions, Israel Sadovnik Socratus for the introduction of the concept of transformation, Cristina Popa-de Greef and Cristian Presura for their useful comment and Sally Rose $(t)$ for the correction of a large part of the manuscript. Special thanks to the Taurida International Symphony Orchestra in 
Saint Petersburg (Russia) under the guidance of Mikhail Golikov, for allowing me to work on this subject while attending their rehearsals, to use the flow of their creative energy.

\section{References}

Backerra, A. C. M. (2010). Uncertainty as a principle. Physics Essays, 23(3), 419-441.

Backerra, A. C. M. (2012). The unification of elementary particles. Physics Essays, 25(4), 601-619.

Backerra, A. C. M. (2014). The quantum-mechanical foundations of gravity. Physics Essays, 27(3), 380-397.

Corry, L. (1998). The influence of David Hilbert and Hermann Minkowski on Einstein's views over the interrelation between physics and mathematics. Endeavor, 22(3), 95-97.

Einstein, A. (1905a). Zur Elektrodynamik bewegter Körper. Annalen der Physik, 17, 891-921

Einstein, A. (1905b). Ist die Trägheit eines Körpers von seinem Energiegehalt abhängig? Annalen der Physik, $18,639-641$.

Einstein, A. (1916). The foundation of the General Theory of Relativity. The collected papers of Albert Einstein, 8, Doc 30, 146-200, Trans. Retrieved from http://hermes.ffn.ub.es/luisnavarro/nuevo_maletin/Einstein GRelativity _1916.pdf

Einstein, A. (1996/1936-1950). The theory of relativity (and other essays). Citadel Press Books, Carol Publishing Group edition.

Elert, G., (1998-2015). The Physics Hypertextbook. http://physics.info/em-spectrum/

Heisenberg, W. (1930/1949). The principles of the quantum theory. New York, NY: Dover publications.

Heisenberg, W. (1971). Schritte über Grenze. München: R. Piper \& Co. Verlag.

Jammer, M. (1974). The Philosophy of Quantum Mechanics. New York, NY: John Wiley and Sons.

Jammer, M. (2000). Concepts of mass in contemporary physics and philosophy. Princeton: Princeton University Press.

Joosten, Y. (2011). Alles über die Zeit. Germany: Marlon Verlag, p. 19.

Messiah, A. (1961). Quantum Mechanics Volume 1. The Netherlands, Amsterdam: North-Holland Publishing Company.

Michelson, A. A., \& Morley, E. W. (1887). On the Relative Motion of the Earth and the Luminiferous Ether. American Journal of Science, 34, 333-345.

Rietdijk, C. W. (2003 March). How do hidden variables fit in natural law? Physics Essays, 16(1), 42-62.

Scientific American. (2014, autumn). A Matter of Time. Volume 23, No. 4.

Smith, H. R. (2008, Sept. 30). What is a black hole? Retrieved from http://www.nasa.gov/audience/forstudents/ $\mathrm{k}-4$ /stories/nasa-knows/what-is-a-black-hole-k4.html

Spitzer, L. Jr. (n.d.). Dying stars. http://www.spitzer.caltech.edu/mission/219-Dying-Stars

The Editors of Encyclopaedia Brittannica. (n.d.). Planck's radiation law. Retrieved from http://www.britannica. $\mathrm{com} /$ science/Plancks-radiation-law

Vaidman, L, (2002). Many-Worlds Interpretation of Quantum Mechanics. Retrieved from http://plato.stanford. edu/entries/qm-manyworlds/

Weizsäcker, C. F. V. (1941). Zur Deutung der Quantenmechanik. Zeitschrift für Physik, 118, 489-509.

Weizsäcker, C. F. V. (1955). Komplementarität und Logik. Die Naturwissenschaften, 42, 521-529, 545-555.

White, H. E. (1964). Introduction to atomic and nuclear physics. New York, NY: Van Nostrand Reinhold Company.

\section{Copyrights}

Copyright for this article is retained by the author(s), with first publication rights granted to the journal.

This is an open-access article distributed under the terms and conditions of the Creative Commons Attribution license (http://creativecommons.org/licenses/by/3.0/). 